\title{
Variability of Near-Field Ground Motion from Dynamic Earthquake Rupture Simulations
}

\author{
by J. Ripperger, P. M. Mai, and J.-P. Ampuero
}

\begin{abstract}
This study investigates near-field ground-motion variability due to dynamic rupture models with heterogeneity in the initial shear stress. Ground velocity seismograms are synthesized by convolving the time histories of slip velocity obtained from spontaneous dynamic rupture models with Green's functions of the medium calculated with a discrete wavenumber/finite-element method. Peak ground velocity (PGV) estimated on the synthetics generally matches well with an empirically derived attenuation relation, whereas spectral acceleration (SA) shows only an acceptable match at periods longer than $1 \mathrm{sec}$. Using the geometric mean to average the two orthogonal components leads to a systematic bias for the synthetics, in particular at the stations closest to the fault. This bias is avoided by using measures of ground motion that are independent of the sensor orientation.

The contribution from stress heterogeneity to the overall ground-motion variability is found to be strongest close to the fault and in the backward directivity region of unilaterally propagating ruptures. In general, the intraevent variability originating from the radiation pattern and the effect of directivity is on the same order or larger than the interevent variability. The interevent ground-motion variability itself is dominated by the hypocenter-station configuration and is influenced only to a lesser extent by the differences in the dynamic rupture process due to the stress heterogeneity. In our modeling approach the hypocenter location is not picked arbitrarily but is determined to be mechanically consistent with the stress heterogeneity through a procedure emulating tectonic stress loading of the fault and nucleation. Compared to the peak ground motion recorded during the 2004 Parkfield, California, earthquake our simulated seismograms show enhanced spatial correlation that may be attributed to the simplicity of the assumed crustal model or to an incomplete representation of the spatial heterogeneity of dynamic rupture parameters. Nevertheless, the intraevent PGV variability in the near-fault region determined for the Parkfield dataset is of the same order of magnitude as for our simulations.
\end{abstract}

\section{Introduction}

Recent, well-instrumented earthquakes generated a large number of ground-motion recordings from sites close to the active fault (e.g., 1999 Chi-Chi, Taiwan, 2000 Tottori, Japan, and 2004 Parkfield, California). In these datasets, the observed variability of ground-motion intensity measures such as peak ground acceleration (PGA) or peak ground velocity (PGV) in the near field is large (e.g., Shakal et al., 2006). This variability potentially originates from differences in the local site conditions close to the recording stations, from varying path effects such as focusing or scattering of the seismic waves and finally from properties of the seismic source itself. This contribution of earthquake source complexity to the ground-motion variability is generally thought to be signifi- cant, especially in the region of less than one or two fault lengths distance.

Early studies on near-source ground motion employed simple theoretical and numerical models to understand its first order characteristics (e.g., Aki, 1968; Haskell, 1969; Archuleta and Frazier, 1978). With increasing computational power and larger number of recordings available, many studies have inverted observed data to construct models of the source process (for a collection of inverted source models, see Mai [2004]). These models are kinematic; that is, they provide a spatiotemporal distribution of the displacements taking place on an earthquake fault that is compatible with the observed ground motion but that does not necessarily 
obey the physical principles of rock fracturing and slip. In contrast, dynamic models attempt to simulate the physical rupture process and the frictional sliding of the rock interfaces past each other. They are typically controlled by initial conditions and a constitutive law relating displacements and stresses on the fault plane. They have been successfully used to model the ground motion of recent earthquakes (e.g., Olsen et al., 1997; Peyrat and Olsen, 2004). Any source inversion, kinematic or dynamic, provides an image of the rupture process of one particular past event.

To estimate seismic hazard in a given region, it is required to anticipate the ground motion due to a future earthquake. To this end, scenario simulations have been performed (e.g., Graves, 1998; Graves and Pitarka, 2004; Olsen et al., 2006) to evaluate ground shaking for specific regions. Only a few studies systematically investigated the influence of different source parameters on the resulting near-source ground motion (e.g., Inoue and Miyatake, 1998; Aagaard et al., 2001, 2004) and even fewer attempt to quantify the uncertainty in the employed source parameters and the associated variability in ground motion. One way to account for the uncertainty in the initial conditions is to parameterize one or more input variables in a stochastic sense (e.g., Oglesby and Day, 2002), perform an ensemble of statistically similar simulations, and evaluate the average prediction and its variability. This approach is expected to play an increasingly important role, in particular due to the advance in computing capabilities (e.g., Olsen et al., 2006).

Recent studies by Ampuero et al. (2006) and Ripperger et al. (2007) explored the stochastic parameterization of initial shear stress for simulations of dynamic earthquake rupture. These papers mainly investigated the modeled rupture process on the fault plane, but because the approach ultimately aims at improving seismic hazard assessment, it is mandatory to verify the ground motions predicted by these simulations against observations. Rather than trying to model particular seismograms of an individual event, we are interested in the general characteristics of near-field ground motion, for example, its peak amplitude and the spatial distribution and variability of these parameters. For observed ground motion, large datasets of these general characteristics have been distilled into empirical attenuation relations. These are essentially equations describing how a measured quantity like PGV is expected to vary with magnitude of an event and observer distance. Often these equations also contain several additional factors to account for the different faulting mechanism and local site response. In this study we investigate how ground-motion intensities of synthetic seismograms for a subset of the dynamic rupture simulations described in Ripperger et al. (2007) compare with recent empirical attenuation relations. An example for such a comparison is provided by Aochi and Douglas (2006), who performed a similar analysis for dynamic rupture models with homogeneous initial stress.

Our study is laid out as follows: In the next section we briefly summarize the approach and the main results of Rip- perger et al. (2007) and describe the selection of the dynamic rupture simulations. The following part describes the setup of the study and the techniques used to compute the synthetic seismograms. The fourth and main part of the article is concerned with the estimation of ground-motion characteristics of engineering interest from these synthetic seismograms and their comparison with empirically derived attenuation relations. In the fifth part we finally investigate how the variability in peak ground motion compares with that of a real event, using the large strong-motion dataset available for the 2004 Parkfield, California, earthquake.

\section{Dynamic Earthquake Rupture Simulations}

The study of Ripperger et al. (2007) focused on exploring statistical descriptions of the initial stress heterogeneity on a fault in order to understand how the stochastic stress parameters control the rupture behavior. Shear stress $\tau_{0}(x, z)$ on the fault plane was modeled as a random field with a normal distribution of values and a given standard deviation. The wavenumber spectra of the stress distributions were constrained to follow a power-law decay at high wavenumbers, similar to the parameterization in the stochastic fault model of Andrews (1980). Based on the spectral characterization of fault slip in kinematic source models (Mai and Beroza, 2002), the wavenumber spectra were set to a constant level below a given corner wavenumber associated with the autocorrelation length $a_{c}$ of the stress field. The Hurst parameter $H$ controlling the fall-off at high wavenumbers was systematically varied, as well as the correlation length $a_{c}$ and the standard deviation (S.D.) of stress.

Apart from the shear stress, the model setup was chosen to be rather simple. Friction on the fault is governed by a linear slip-weakening constitutive relation, where the yield strength $\tau_{s}$ and frictional sliding strength $\tau_{d}$ as well as the critical slip-weakening distance $D_{c}$ are uniform. Thus, the fracture energy $G_{c}=1 / 2 D_{c}\left(\tau_{s}-\tau_{d}\right)$ is also uniform. The fault itself is planar and is embedded in a homogeneous elastic full space. The assumption of a full space for the dynamic simulations is justifiable for buried faults (depth $>3 \mathrm{~km}$ ); hence, our subsequent computations of ground motion assume a homogeneous half-space with the top edge of the fault at 5-km depth (see the section Fault and Receiver Geometry). Our modeling assumes that tectonic loading occurs as uniformly increasing shear stress on the fault plane, raising the initial stress field to a critical state. This critical stress state, which is used as the starting stress field in the dynamic rupture simulations, is found through the approximate procedure described in detail in Ripperger et al. (2007) or through the complete procedure described in the Appendix. Rupture nucleation thus does not occur at a specified location where an instability is forced to grow by adjusting stress and friction values. Instead, our method ensures that the hypocenter for each rupture event is determined in a physically self-consistent manner for the underlying heterogeneous stress field and our assumptions on the loading and 
nucleation process. The setup for the dynamic simulations is well suited to being treated numerically by a boundary integral method. The simulations were performed using the code MDSBI developed by Dunham (2005) using the methodology of Geubelle and Rice (1995). The detailed model parameters are listed in Table 1.

The S.D. of the stress field was found to exert the strongest influence on the overall characteristics of the rupture because it mainly controls the average level of stress $\bar{\tau}_{0}$ at the beginning of the rupture. The dimensionless $\overline{\tau_{0}}$ is defined as $\overline{\tau_{0}}=\left\langle\left(\tau_{0}-\tau_{d}\right) /\left(\tau_{s}-\tau_{d}\right)\right\rangle$, where angle brackets denote averaging across the fault plane. Note that $\overline{\tau_{0}}$ is related to the dimensionless parameter $S=\left(\tau_{s}-\tau_{0}\right) /\left(\tau_{0}-\tau_{d}\right)$ as used by Das and Aki (1977) and others through $\overline{\tau_{0}}=$ $\langle 1 /(1+S)\rangle$, where angle brackets again denote averaging over the fault plane. For considering averaged values, we favor the use of $\overline{\tau_{0}}$ over $S$, because it has the advantageous property of scaling linearly with initial stress $\tau_{0}$ and does not have a singularity at $\tau_{0}=\tau_{d}$. A threshold in the average stress level was identified above which ruptures tend to become unstoppable by the stress heterogeneity itself.

For the computation of ground motion we selected a subset of the large collection of dynamic rupture simulations of Ripperger et al. (2007). First of all, we restrict the analysis to a range of seismic moments $M_{\mathrm{w}} 6.7-6.9$. In this range the order of magnitude of the chosen fracture energy value $G=$ $0.9 \mathrm{MJ} / \mathrm{m}^{2}$ is in agreement with empirical estimates of fracture energy (e.g., Abercrombie and Rice, 2005; Mai et al., 2006). Secondly, only events are considered that do not exhibit wide-spread supershear rupture velocity. This was achieved by excluding events with average rupture velocity above 0.8 times the shear-wave velocity. In total these criteria yielded 61 events out of a suite of more than 400 simulated ruptures. Their final slip distributions and rupture front contours are displayed in Figure 1, and their average macroscopic rupture properties are summarized in Table 2 for each set of stress field parameters $H, a_{c}$, and S.D. The properties of the selected dynamic events are consistent with empirically derived source scaling laws (e.g., Wells and Coppersmith, 1994; Mai and Beroza, 2000). While this is

\section{Table 1}

Model Parameters of All Dynamic Rupture Simulations of Ripperger et al. (2007) Studied in This Article

\begin{tabular}{lcc}
\hline \multicolumn{1}{c}{ Parameter } & Symbol & Value \\
\hline Density & $\rho$ & $2800 \mathrm{~kg} / \mathrm{m}^{3}$ \\
$P$-wave velocity & $v_{p}$ & $6000 \mathrm{~m} / \mathrm{sec}$ \\
$S$-wave velocity & $v_{s}$ & $3464 \mathrm{~m} / \mathrm{sec}$ \\
Critical slip-weakening distance & $D_{c}$ & $0.2 \mathrm{~m}$ \\
Yield strength & $\tau_{s}$ & $24 \mathrm{MPa}$ \\
Frictional sliding strength & $\tau_{d}$ & $15 \mathrm{MPa}$ \\
Grid spacing & $\Delta x$ & $150 \mathrm{~m}$ \\
Time sampling & $\Delta t$ & $0.013 \mathrm{sec}$ \\
Fault dimensions & & $15 \times 30 \mathrm{~km}$ \\
\hline
\end{tabular}

The same density and seismic velocity values are used for the computation of synthetic ground motion. also true for the ratio $E_{r} / M_{0}$ of radiated energy to seismic moment, we note that $E_{r} / M_{0} \approx 1-4 \times 10^{-5}$ obtained for our simulations is in the lower range of values estimated for similar sized real earthquakes (e.g., Kanamori and Brodsky, 2004). This likely indicates that real events can exhibit largescale fluctuations in their rupture propagation velocity that are more pronounced than the variations present in our dynamic simulations (Fig. 1).

All selected rupture models have the same stress field correlation length $a_{c}=5 \mathrm{~km}$. Because of differences in the Hurst exponent $H$ the initial stress fields differ in their high-wavenumber contents. This can be seen in Figure 1, where the rupture front contours of the models with Hurst exponent $H=1$ (models 36-61) look smoother than those of the models with $H=0$ and $H=0.5$. However, as noted by Ripperger et al. (2007), differences in high-wavenumber variability do not strongly influence the overall rupture behavior, which is mainly determined by the average stress level $\overline{\tau_{0}}$. With average stress levels of $\overline{\tau_{0}}=0.35-0.60$ all selected events are located above the size transition discussed in Ripperger et al. (2007); that is, their final size is mainly determined by the maximum fault extensions allowed in the dynamic simulations as reflected in their very similar rupture areas. The differences in the average stress level result in differences in the amount of slip on the fault and therefore in the moment magnitudes $M_{\mathrm{w}}$. The aforementioned selection criteria ensure that these variations in the macroscopic rupture properties remain within certain limits that allow all the selected models to be reasonably well considered as realizations of the same earthquake. Furthermore, in the comparison to empirical attenuation relations in the main part of this study, we will mainly analyze the residuals, that is, the differences between each model and the corresponding empirical estimate for its particular moment magnitude. Because this effectively evens out the differences of average stress level, the main differences between the models are their hypocenter location and the relative distribution of the highand low-stress patches.

The hypocenter-station configuration is expected to be responsible for a significant part of the ground-motion variability (e.g., Aagaard et al., 2001). Therefore, in addition to the simulations selected from Ripperger et al. (2007), we performed 30 new simulations in which the initial stress field was shifted to make the hypocenters coincide at the same point at $z=15 \mathrm{~km}$ and $x=-10 \mathrm{~km}$ (Fig. 2). Hence, the ground-motion variability of these 30 rupture models originates solely from the different random phases of their initial stress fields, which will allow us to separately study this source of variability. The 30 new simulations were performed for only one set of stress field parameters $(H=0.5$, $a_{c}=5 \mathrm{~km}$, and S.D. $=2 \mathrm{MPa}$ ) with their average macroscopic source properties listed in Table 2. They differ from the previous simulations in the way the static stress loading and nucleation is computed. In contrast to the approximate nucleation scheme employed in Ripperger et al. (2007), the new procedure (see the Appendix) includes the effect of 

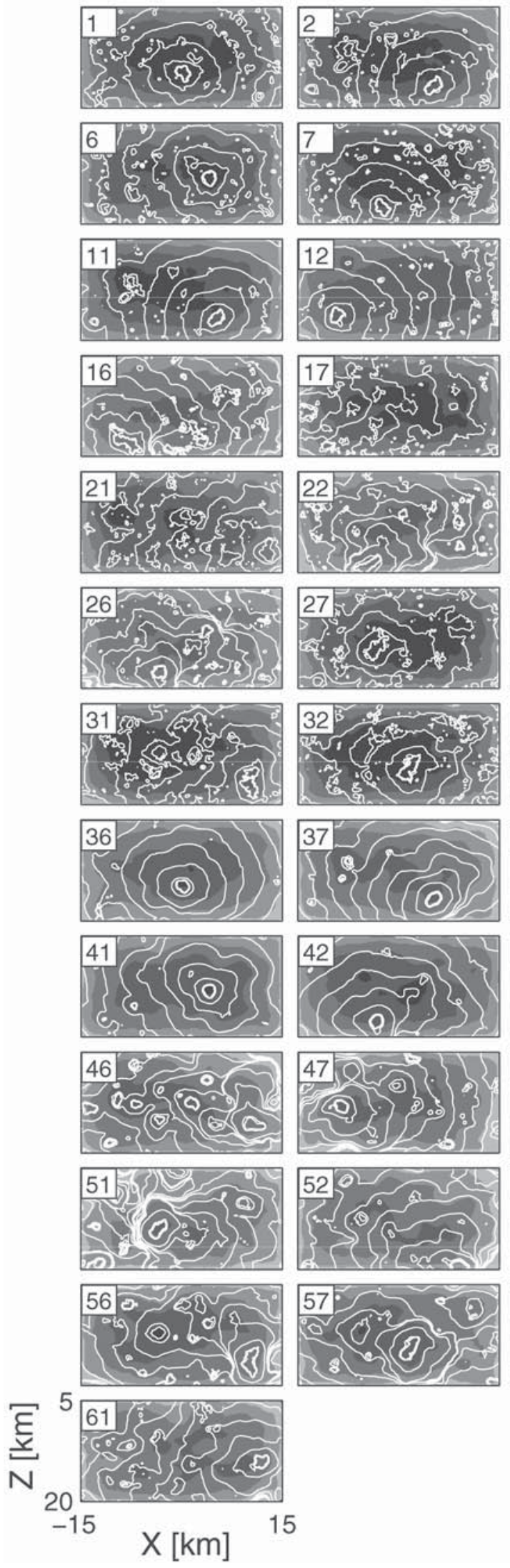

15
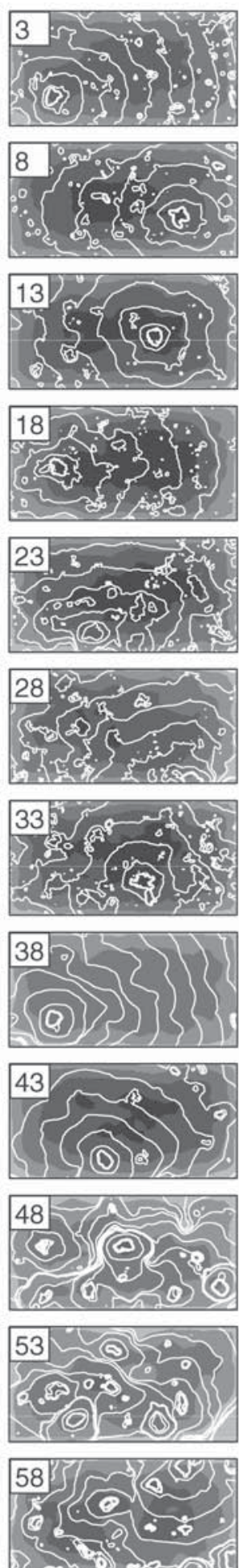

B. .

Figure 1. Final slip distributions of all runs and contours of the rupture front every second. 
Table 2

Stress Field Parameters and Averaged Macroscopic Properties of the Dynamic Rupture Simulations

\begin{tabular}{cccccccc}
\hline$H$ & $a_{c}(\mathrm{~km})$ & S.D. (MPa) & $\bar{\tau}_{0}$ & $M_{\mathrm{w}}$ & Area $\left(\mathrm{km}^{2}\right)$ & Number & $N$ \\
\hline \multicolumn{7}{c}{61 events } & with variable hypocenters: \\
0.0 & 5.0 & 3.0 & 0.54 & 6.87 & 450 & $1-9$ & 9 \\
0.5 & 5.0 & 2.0 & 0.53 & 6.87 & 450 & $10-15$ & 6 \\
0.5 & 5.0 & 3.0 & 0.48 & 6.83 & 450 & $16-35$ & 20 \\
1.0 & 5.0 & 2.0 & 0.44 & 6.81 & 450 & $36-45$ & 10 \\
1.0 & 5.0 & 3.0 & 0.38 & 6.76 & 447 & $46-61$ & 16
\end{tabular}

30 events with fixed hypocenters:

\begin{tabular}{llllllll}
0.5 & 5.0 & 2.0 & 0.45 & 6.80 & 450 & Fig. 2 & 30 \\
\hline
\end{tabular}

The 61 simulations with variable hypocenters were selected from Ripperger et al. (2007) while the 30 simulations with fixed hypocenters have been newly performed for this study. Number refers to the event numbers in Figure 1, and $N$ gives the total number of selected simulations with the given parameter set $H, a_{c}$, and S.D.

(a)
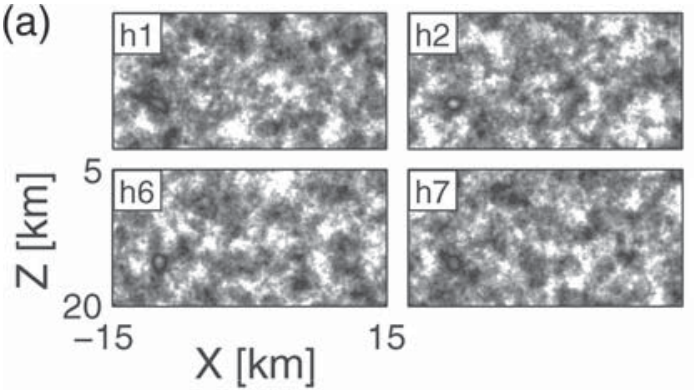

5
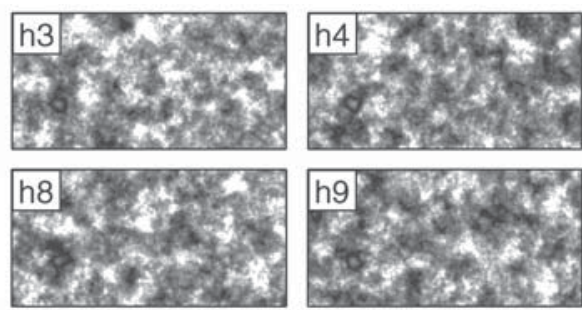
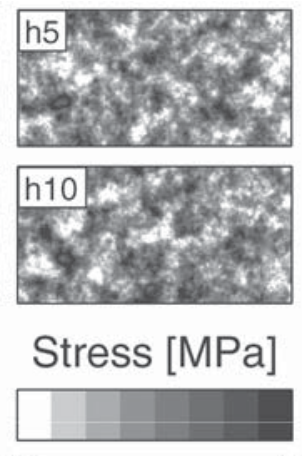

15
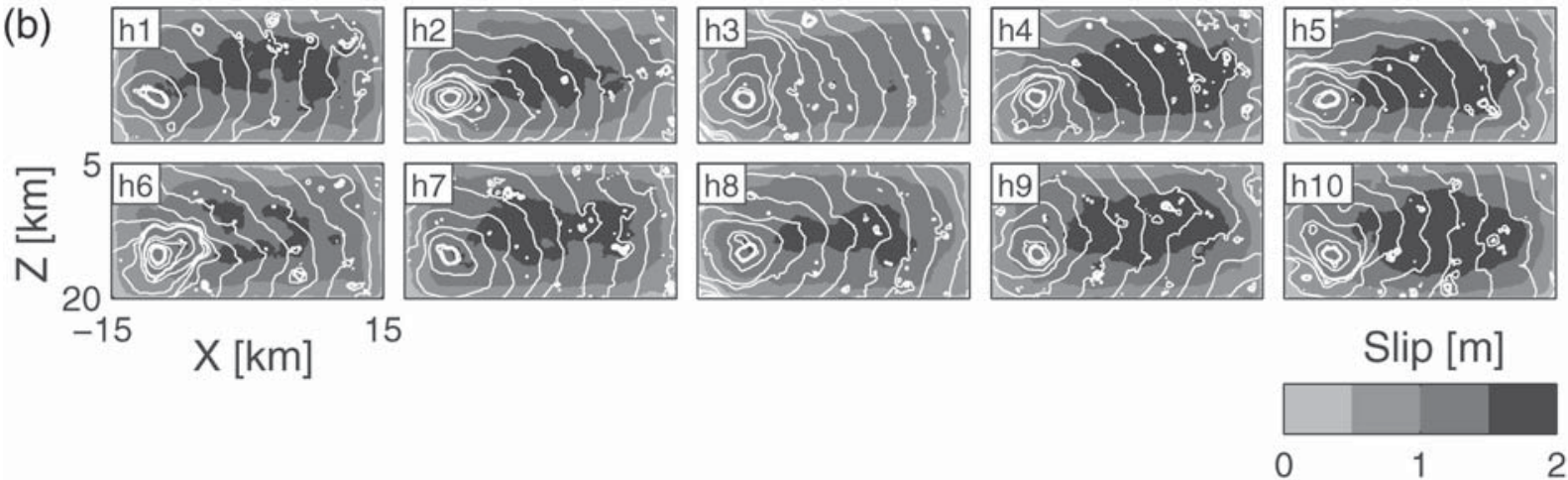

(c)
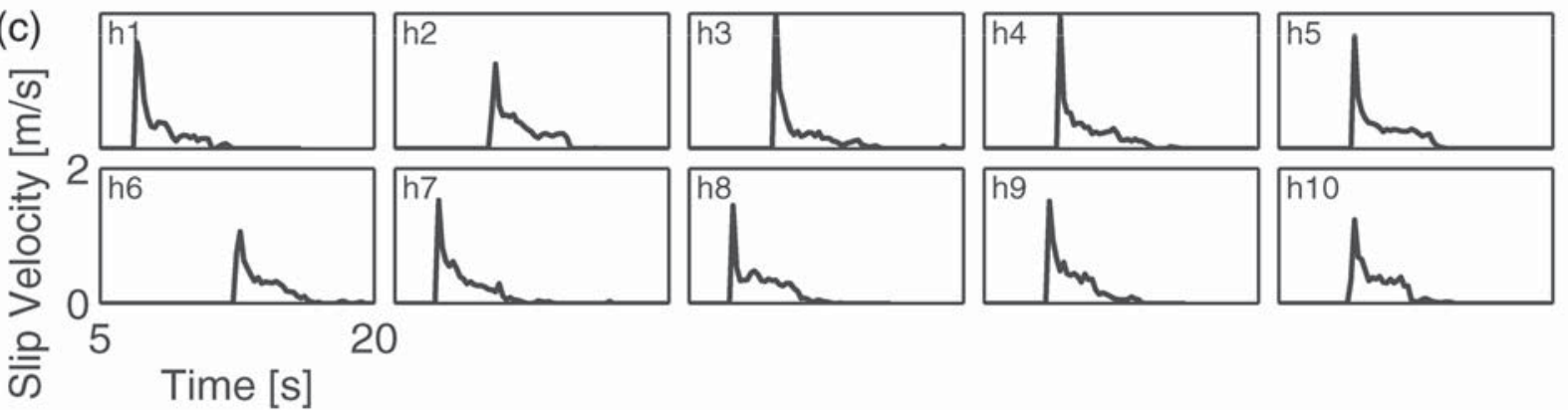

20

Figure 2. Initial stress distributions and resulting final slip distributions of 10 examples out of the 30 runs with hypocenters fixed at $x=-10 \mathrm{~km}, z=15 \mathrm{~km}$. Overlain on the slip distributions are the contours of the rupture front every second. The lowermost panels show slip-velocity functions at the center of the fault. Simulation numbers preceded by an "h" connect the rupture models to the example seismograms in Figure 7. 
quasi-static preslip inside the nucleation zone. However, the differences during the nucleation phase are not expected to significantly alter the results in terms of the peak ground motions.

\section{Computation of Synthetic Ground Motion}

During the simulations of dynamic rupture propagation described in the preceding discussion, the time histories of slip-velocity have been stored for each grid point of the fault. These slip-velocity traces are then convolved with Green's functions of the medium for a desired fault-receiver geometry to obtain synthetic seismograms of ground velocity.

\section{Fault and Receiver Geometry}

Our synthetic ruptures are assumed to be strike-slip events taking place on a vertical fault plane. Because the dynamic rupture simulations were performed for a homogeneous full space, we place the top of the fault at $5-\mathrm{km}$ depth. For more shallow faulting the influence of the free surface on the dynamic propagation might become significant, but for depths larger than $5 \mathrm{~km}$ this effect is thought to be negligible (see, e.g., Nielsen and Olsen [2000] and Oglesby et al. [2000] for the case of thrust faulting).

Seismograms were computed for a set of 50 hypothetical stations surrounding the fault at various azimuths and distances. The particular receiver configuration was chosen to sample the distance range between 1 and $60 \mathrm{~km}$ in terms of the closest distance to the surface projection of the fault (commonly denoted as the Joyner-Boore distance $r_{j b}$ ) with approximately equal azimuthal coverage. We positioned receivers along lines running parallel to the fault at various distances. In addition, beyond the ends of the fault receiver profiles extend radially outward with azimuths of $0^{\circ}, 30^{\circ}$, and $60^{\circ}$. The receiver locations are specified in Table 3 and illustrated in Figure 3. Note that the empirical attenuation relations we compare our results against are provided as functions of distance to the rupture plane $r_{\text {rup }}$ and distance to the seismogenic part of the rupture plane $r_{\text {seis }}$. With our chosen setup, these two distance metrices are identical and can be simply expressed as

$$
r_{\text {rup }}=r_{\text {seis }}=\sqrt{r_{j b}^{2}+(5 \mathrm{~km})^{2}} .
$$

Because large earthquakes are more likely to nucleate in the deeper part of the seismogenic zone (e.g., Mai et al., 2005), we only allow hypocenters to be located in the lower half of the fault plane, that is, between $12.5-$ and $20-\mathrm{km}$ depth. For all events that originally had shallower hypocenters, we flipped the whole event upside down, which is possible because the dynamic rupture simulation was performed for a full space. The obtained hypocenter distribution is illustrated in fault-plane view in Figure 4.

As mentioned previously, to single out the groundmotion variability due to the initial stress heterogeneity only, we computed 30 additional models, where the hypocenter was fixed at $z=15 \mathrm{~km}$ and $x=-10 \mathrm{~km}$, which makes the ruptures propagate primarily unilateral.

\section{Green's Functions}

To calculate the Green's functions we utilize the discrete wavenumber/finite-element method (DWFE) by Olson et al. (1984) as implemented in the COMPSYN package by Spudich and Xu (2003). The codes in this package make use of the reciprocity theorem and provide the Green's functions in the form of tractions on a fault plane resulting from a delta pulse at the receiver location. These tractions $\tau_{x x}, \tau_{x y}$, and $\tau_{x z}$ are specified in the frequency domain and on an irregular spatial grid that varies for different frequencies. We therefore interpolate the traction values on a rectangular grid and subsequently perform the inverse Fourier transform to retrieve traction time histories at the same points of the fault

Table 3

Coordinates of Receiver Locations at which Ground Motions are Computed

\begin{tabular}{|c|c|c|c|c|c|c|c|c|c|}
\hline \multicolumn{2}{|c|}{$r_{j b} \approx 1 \mathrm{~km}$} & \multicolumn{2}{|c|}{$r_{j b} \approx 3 \mathrm{~km}$} & \multicolumn{2}{|c|}{$r_{j b} \approx 10 \mathrm{~km}$} & \multicolumn{2}{|c|}{$r_{j b} \approx 30 \mathrm{~km}$} & \multicolumn{2}{|c|}{$r_{j b} \approx 60 \mathrm{~km}$} \\
\hline$x$ & $y$ & $x$ & $y$ & $x$ & $y$ & $x$ & $y$ & $x$ & $y$ \\
\hline 16.0 & 0.3 & 18.0 & 0.3 & 25.0 & 0.3 & 45.0 & 0.3 & 75.0 & 0.3 \\
\hline 15.9 & 0.5 & 17.6 & 1.5 & 23.5 & 5.0 & 41.0 & 15.0 & 67.0 & 30.0 \\
\hline 15.5 & 0.9 & 16.5 & 2.6 & 20.0 & 8.5 & 30.0 & 26.0 & 45.0 & 52.0 \\
\hline 15.0 & 1.0 & 15.0 & 3.0 & 15.0 & 10.0 & 15.0 & 30.0 & 15.0 & 60.0 \\
\hline 7.5 & 1.0 & 7.5 & 3.0 & 7.5 & 10.0 & & & & \\
\hline 0.0 & 1.0 & 0.0 & 3.0 & 0.0 & 10.0 & 0.0 & 30.0 & & \\
\hline-7.5 & 1.0 & -7.5 & 3.0 & -7.5 & 10.0 & & & & \\
\hline-15.0 & 1.0 & -15.0 & 3.0 & -15.0 & 10.0 & -15.0 & 30.0 & -15.0 & 60.0 \\
\hline-15.5 & 0.9 & -16.5 & 2.6 & -20.0 & 8.5 & -30.0 & 26.0 & -45.0 & 52.0 \\
\hline-15.9 & 0.5 & -17.6 & 1.5 & -23.5 & 5.0 & -41.0 & 15.0 & -67.0 & 30.0 \\
\hline-16.0 & 0.3 & -18.0 & 0.3 & -25.0 & 0.3 & -45.0 & 0.3 & -75.0 & 0.3 \\
\hline
\end{tabular}

Coordinates listed are measured in kilometers and are sorted by the closest distance to surface projection of the fault plane, commonly known as the Joyner-Boore distance $r_{j b}$. Also see Figure 3 for a map of the receivers. 

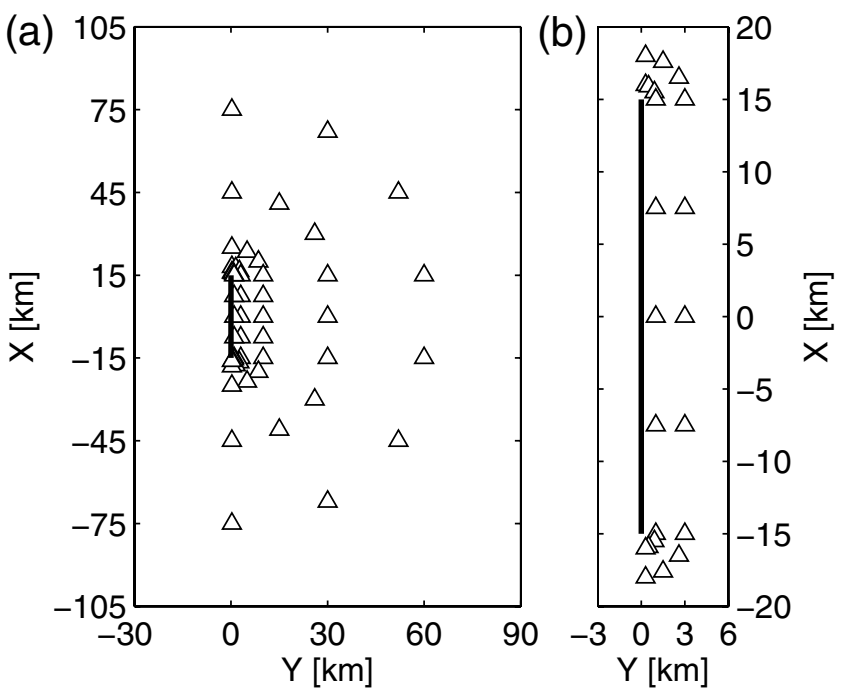

Figure 3. Map-view of station configuration. Thick line indicates the surface projection of the fault. The right panel (b) displays a zoom-in on the fault. The site distances from the surface projection of the fault are 1,3,10,30, and $60 \mathrm{~km}$. Receiver coordinates are specified in Table 3.

where slip-velocity traces are available from the dynamic simulations.

The three components of ground velocity $v_{x}, v_{y}$, and $v_{z}$ at each receiver site are obtained by convolving the slipvelocity signal $\Delta \dot{u}(t)$ with the corresponding traction time history at each point $(i, j)$ on the fault and finally summing the contribution of all grid points:

$$
\begin{aligned}
& v_{x}(t)=\sum_{i j} \Delta \dot{u}(t, i, j) * \tau_{x x}(t, i, j), \\
& v_{y}(t)=\sum_{i j} \Delta \dot{u}(t, i, j) * \tau_{x y}(t, i, j), \\
& v_{z}(t)=\sum_{i j} \Delta \dot{u}(t, i, j) * \tau_{x z}(t, i, j),
\end{aligned}
$$

where $*$ denotes time convolution. Note, that only the $x$ component (the along-strike component) of slip velocity is used for the computation of ground motion although in the dynamic simulations the rake angle had been allowed to vary. But because the initial shear stress was nonzero only for the $x$ component, the amplitude of the perpendicular down-dip component of slip velocity remained at negligible levels. The COMPSYN package allows for computing Green's functions for an arbitrary 1D velocity-density structure. However, we restrict the comparison to the simplest case of a homogeneous half-space with the same medium parameters as used in the dynamic rupture simulations (Table 1). The Green's functions are designed to be accurate up to a frequency of $4 \mathrm{~Hz}$. The time series are computed for a maximum duration of $45 \mathrm{sec}$ with a time sampling of $0.0216 \mathrm{sec}$. The dynamic rupture simulations had been computed with a timestep of $0.013 \mathrm{sec}$, but the slip-velocity values had been

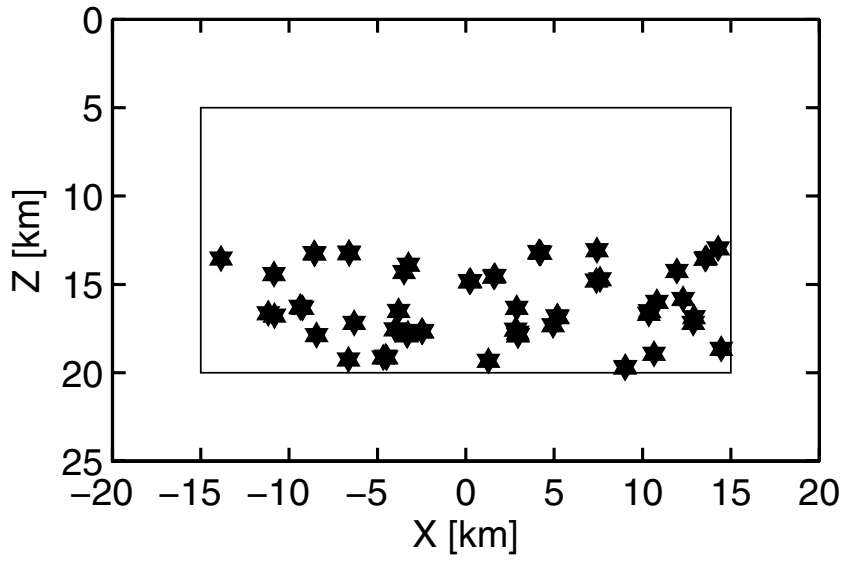

Figure 4. Side view of the fault plane (black rectangle) and the locations of the hypocenters (black stars).

written to file only every fifteenth timestep with a time sampling of $0.195 \mathrm{sec}$ and a Nyquist frequency of roughly $2.5 \mathrm{~Hz}$. The slip-velocity time histories are linearly interpolated to the same time sampling as the traction time series before convolution of the two time series in the Fourier domain. Because of the reduced sample spacing of the slipvelocity functions from the dynamic simulations, the synthetic velocity seismograms are accurate up to only about $2.5 \mathrm{~Hz}$. Prior to using the synthetic seismograms for subsequent analyses, we therefore low-pass filter with a fourth order Butterworth filter with a cutoff frequency of $2.5 \mathrm{~Hz}$. One disadvantage of the COMPSYN package is that the Green's functions are computed for a fully elastic medium and, therefore, do not include inelastic attenuation. One way to approximately account for attenuation is to subsequently filter the seismograms with a $t^{*}$-operator (Futterman, 1962), which is dependent on travel time and the $Q$-value of the medium. We have performed this filtering for a number of $Q$-values, taking $r_{j b} / v_{s}$ as a proxy for the direct shear-wave travel time. We will show some results in the section on PGV, but where not explicitly noted otherwise, the results presented in this article are obtained without this additional filtering.

\section{Ground-Motion Characteristics}

For seismic hazard assessment and engineering purposes many different attributes of ground-motion records have been measured to express their intensity and damage potential. The most commonly used are PGA and spectral acceleration (SA) at different periods. But recently the destructive potential of velocity pulses has been acknowledged more widely (e.g., Wald et al., 1999; Boatwright et al., 2001), and PGV has received increased attention. For all these measures of ground-motion intensity large datasets have been compiled from recorded seismograms, and researchers have derived empirical attenuation relations that essentially describe the decay of shaking level with distance from the earthquake source. 
In the following sections we will present examples of our synthetic waveforms and compare the measures of ground-motion intensity and their variability to recent empirical attenuation relations.

\section{Example Waveforms}

Two typical examples of simulations and their associated synthetic velocity seismograms are presented in Figures 5 and 6. Figure 5 shows a bilateral event (number 10 in Fig. 1) starting approximately at the center of the fault. Accordingly, the seismograms in both rupture propagation directions are very similar in shape and amplitude. The faultparallel component is largest at the receivers located in the direction perpendicular to the fault, while the fault-normal component exhibits the largest amplitudes at stations in both directions along the fault strike. Both features are expected from the $S$-wave radiation pattern of a double-couple source (e.g., Aki and Richards, 2002, p. 81).

The predominantly unilateral rupture propagation of event number 12 is illustrated in Figure 6. The signature of the unilateral propagation (e.g., Somerville et al., 1997) can clearly be seen in the seismograms. A strong velocity pulse is visible in the fault-normal component in the forward directivity direction, its amplitude exceeding that of the bilateral case. No such pulse is apparent in the backward di- rectivity direction, where the amplitudes are generally lower than in the bilateral case.

Figure 7 displays seismogram examples for 10 different realizations of the initial stress field (labeled h1 through h10) with fixed hypocenter. Shown are seismograms of faultnormal velocity at three receivers in $1-\mathrm{km}$ distance from the surface projection of the fault trace. One receiver is located at the center of the fault $(x=0)$ and the other two are located at each end of the fault trace $(x=-15$ and 15). Again the directivity effect is clearly visible as generally higher amplitudes of the velocity pulses in the forward directivity position. Because the hypocenter was identical for these simulations, the differences in the seismogram waveforms and peak amplitudes originate solely from differences in the rupture propagation due to the heterogeneous stress distributions. However, as will be discussed in the following, the variability in peak amplitudes due to the directivity effect is in most cases larger than the variability due to the stress heterogeneity.

\section{Peak Ground Velocity}

PGV is the most preferable measure to estimate on our synthetic seismograms, because it is sensitive to the frequency range that is covered by the rupture simulations. In contrast, PGA in real data is typically associated with higher frequencies that are not accurately resolved in our
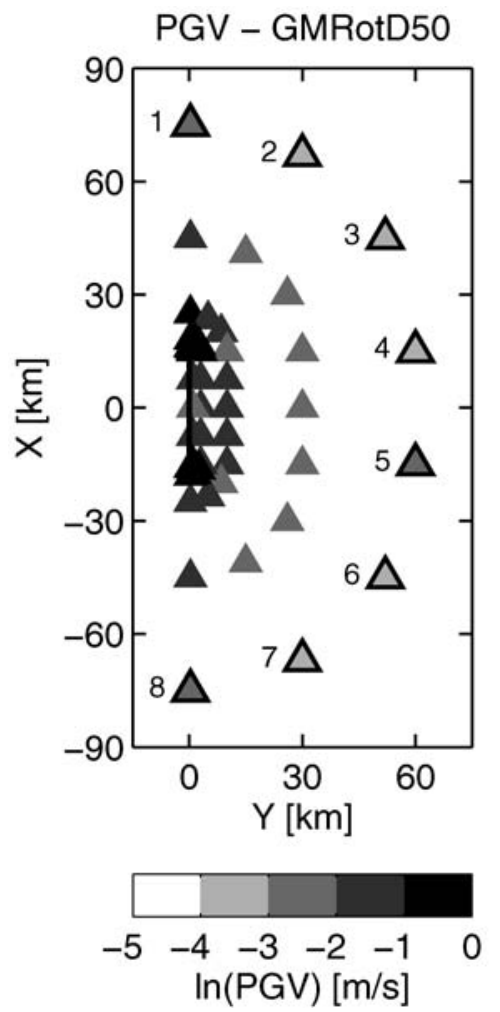
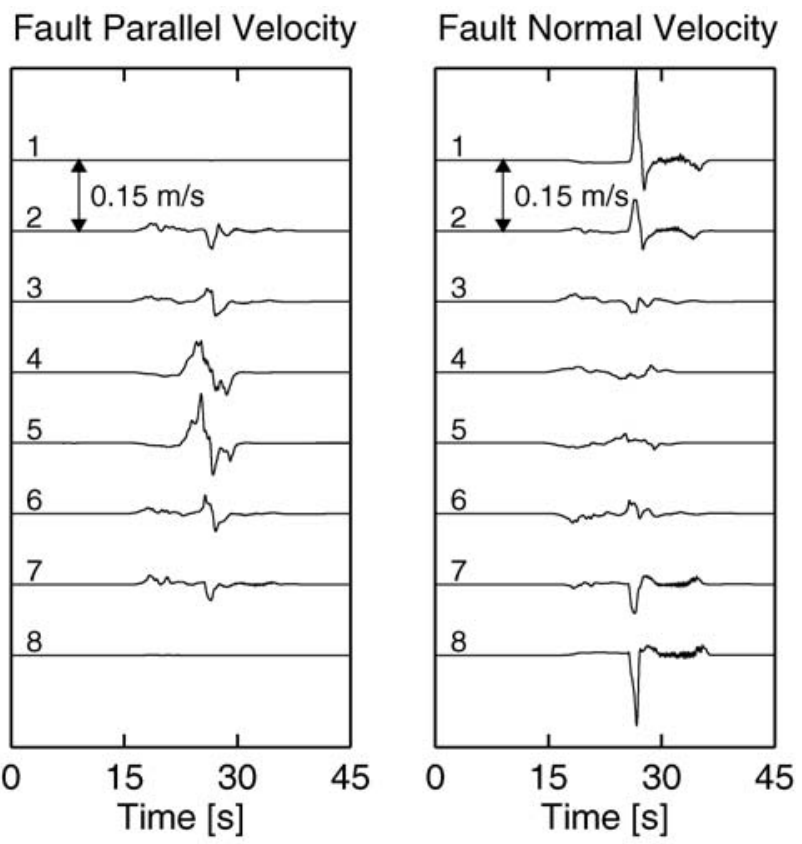

Event \#10 - Bilateral

Figure 5. Map of horizontal PGV values (GMRotD50) for a single bilateral event (number 10 in Fig. 1), along with seismograms of the receiver ring at $r_{j b} \approx 60 \mathrm{~km}$. Note the pronounced velocity pulses on the fault-normal component due to the directivity effect. Because of the bilateral rupture propagation, their amplitude is on the same order of magnitude in both (positive and negative $x$ ) directions from the fault. 

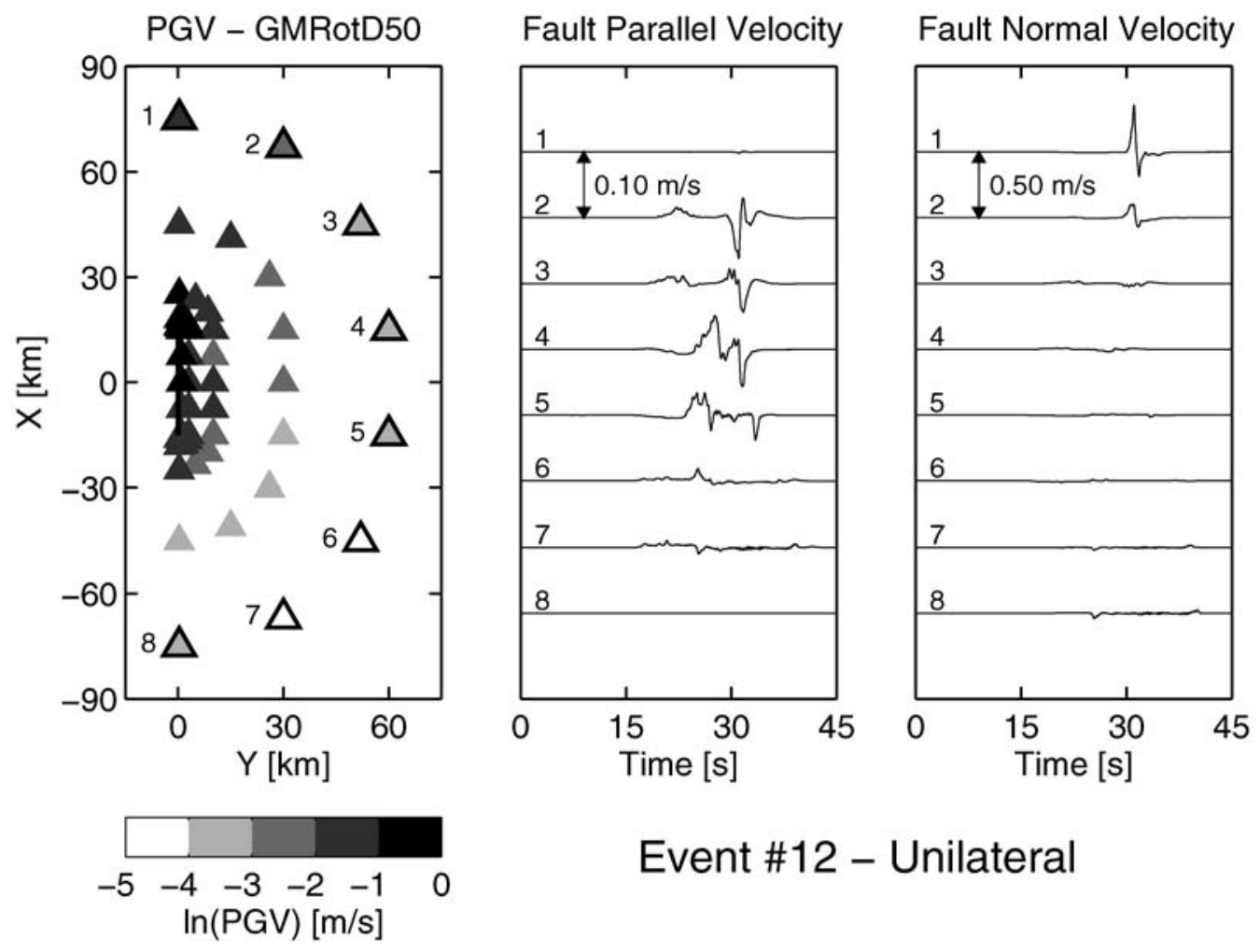

\section{Event \#12 - Unilateral}

Figure 6. Map of horizontal PGV values (GMRotD50) for a single unilateral event, along with seismograms of the receiver ring at $r_{j b} \approx 60 \mathrm{~km}$. The rupture is propagating in the positive $x$ direction and thereby generates a strong velocity pulse on the fault-normal component at receivers in the forward directivity region. The amplitude of this directivity pulse is higher than for the bilateral rupture (Fig. 5). Note the different scale of the seismogram axes for the two components.

synthetics, which are limited to a maximum frequency of $2.5 \mathrm{~Hz}$.

We compare our results to the attenuation relations for PGV derived by Campbell (1997, 2000, 2001). These relations give $\mathrm{PGV}$ as a function of distance of the receiver to the seismogenic rupture plane $r_{\text {seis }}$, which we simply take as the distance to the top of the fault plane at $5 \mathrm{~km}$. Furthermore the relations are parameterized in terms of moment magnitude $M_{\mathrm{w}}$, style of faulting, and local site geology. For a first comparison we set $M_{\mathrm{w}}$ to 6.8 and set the appropriate factors for pure strike-slip faulting and a hard-rock site condition, because this site class comes closest to our assumed homogeneous half-space. One issue that deserves careful consideration for the comparison of horizontal PGV is the way the two horizontal components of ground velocity are treated. This is illustrated in Figure 8. Separate PGV estimates of the fault-parallel and fault-normal component are presented in panels a and $b$, respectively, of Figure 8. The fault-parallel component shows a large variability, mainly resulting from the receivers in approximately nodal positions of the radiation pattern, that is, close to the continuation of the fault trace along strike. For his regression analysis Campbell (1997) used the geometric mean of the separately derived PGV values as depicted in Figure 8c. At larger distances, the average PGV values agree very well with the empirical attenuation curve. At the shortest distance the aver- age PGV value seems to decrease. This feature is only an effect of the orientation of the two components of the receiver. It vanishes for other estimates of horizontal PGV that are independent of receiver orientation such as the maximum amplitude $\max \left(\sqrt{v_{x}^{2}+v_{y}^{2}}\right)$ displayed in Figure $8 \mathrm{~d}$. This is a physically more reasonable measure, because it represents a peak in horizontal velocity occurring at a single point in time. In contrast, the geometric mean of the two PGV components can originate from two separate wave arrivals in the two components at different times and is generally strongly dependent on the particular receiver orientation. Because the maximum amplitude as defined previously is always equal to or larger than the geometric mean of the separate PGV estimates, the obtained average PGV values are well above the empirical relation.

The issue of sensor orientation has recently been addressed by Boore et al. (2006) who propose the orientationindependent measure GMrotD50. It comprises a rotation of the two orthogonal components from $1^{\circ}$ to $90^{\circ}$ in $1^{\circ}$ steps and an evaluation of the geometric mean for each pair of rotated times series. The final measure is the median value of all 90 values of the geometric mean. The resulting PGV values are depicted in Figure 8e. An empirical attenuation relation of this measure is not yet available, but Beyer and Bommer (2006) investigated the relationship between GMrotD50 and empirical attenuation curves based on the geometric mean 
(a) Forward Directivity

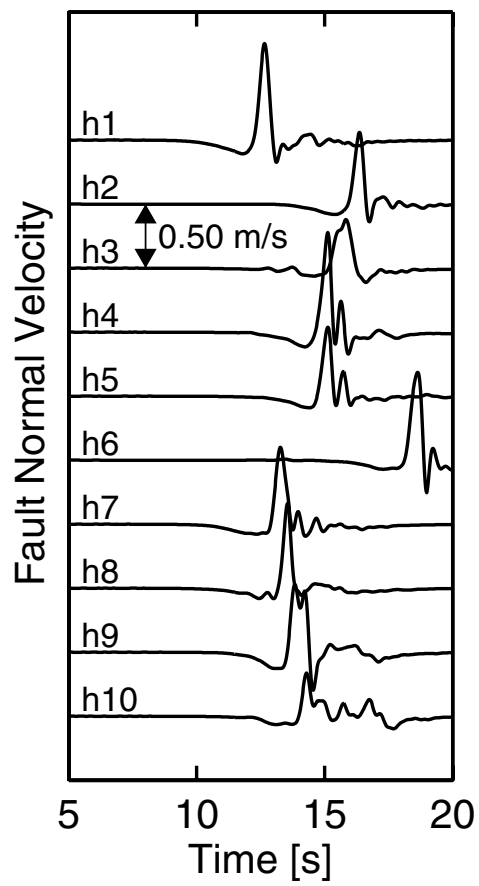

(b) Fault Center

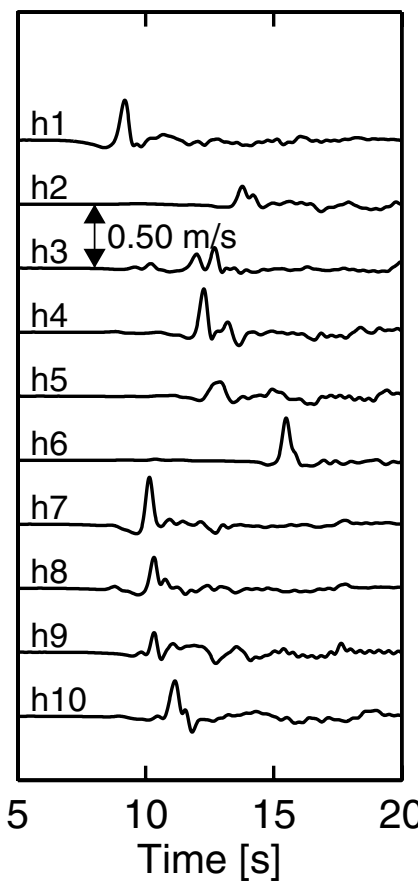

(c) Backward Directivity

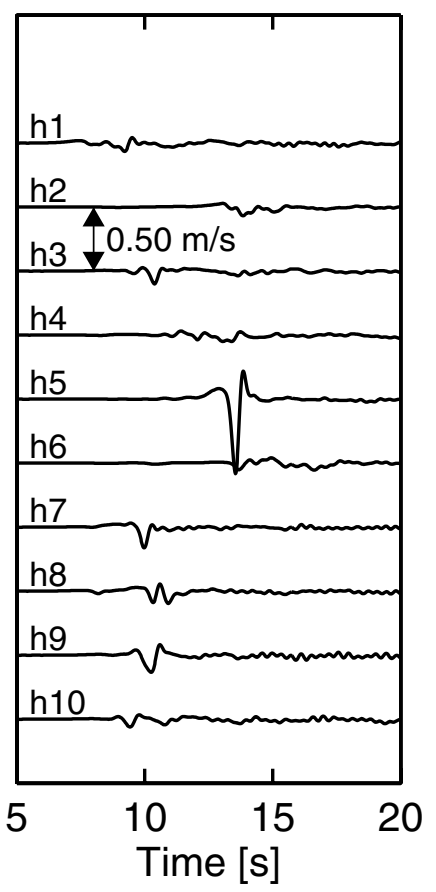

Figure 7. Example seismograms for the 10 different realizations of the initial stress field with fixed hypocenters shown in Figure 2 (labels $\mathrm{h} 1$ through h10 at the traces correspond to those of the rupture models in Fig. 2) at three receiver locations in Joyner-Boore distances of $1 \mathrm{~km}$. Only the fault-normal component of ground velocity is shown. (a) Seismograms at a receiver in the forward directivity region $(x=15 \mathrm{~km}$, $y=1 \mathrm{~km})$. (b) Seismograms at a receiver located at a central position $(x=0 \mathrm{~km}, y=1 \mathrm{~km})$. (c) Seismograms at a receiver in the backward directivity region $(x=-15 \mathrm{~km}, y=1 \mathrm{~km})$.

definition, finding only minor differences. Thus GMRotD50 constitutes a better measure than the geometric mean to compare the empirical data (with typically random sensor orientation) and our simulations (with components oriented exactly parallel and normal to the fault plane) and will be used throughout the remainder of this article.

A difference observable in Figure 8e is the lower fall-off rate with distance of our PGV estimates compared to the empirical relation, which is potentially caused by the omission of inelastic attenuation. The effect of applying the attenuation correction via the $t^{*}$-operator (Futterman, 1962) is shown in Figure $8 \mathrm{~d}$ for $Q=100$. For assumed values of $Q=$ 200 or larger (not shown), appropriate for average crustal rocks, the difference compared to the purely elastic case is very small. A slight reduction of PGV values is observed for $Q=100$, where the fall-off with distance of our synthetic $\mathrm{PGV}$ values is comparable with the empirical attenuation relation and the mean PGV value is within the $1-\sigma$ bounds of the empirical attenuation relation. All further results presented in this article were obtained without applying the attenuation correction, unless explicitly noted otherwise.

\section{PGV Variability}

Having confirmed the overall agreement of our PGV estimates with the empirical attenuation relation, we now proceed to a closer inspection of the variabilities. Part of the variability in the PGV values plotted in Figure 8 is due to the differences in moment magnitude $M_{\mathrm{w}}$ 6.7-6.9 of the events compared to the assumed $M_{\mathrm{w}} 6.8$ for the attenuation relation. To compare each PGV value with the appropriate value predicted from the attenuation relations, we define the residual $r$ as

$$
r=\ln \left(\mathrm{PGV}_{\mathrm{syn}}\right)-\ln \left(\mathrm{PGV}_{\mathrm{emp}}\right)=\ln \left(\frac{\mathrm{PGV}_{\mathrm{syn}}}{\mathrm{PGV}_{\mathrm{emp}}}\right)
$$

where $\mathrm{PGV}_{\text {syn }}$ and $\mathrm{PGV}$ emp are the PGV values from the synthetic seismograms and the empirical prediction, respectively. Figure 9 presents the distribution of these residuals separated for each distance range. The mean value of the residuals is always positive and generally increasing with distance. This reflects the slight overprediction of PGV values with increasing distance as noted previously. The standard deviation $\sigma_{r}$ of the residuals remains approximately constant at $\sigma_{r}=0.54-0.62$ for all distances (Table 4). This variability is somewhat larger than the standard deviations of 0.39-0.55 specified for the empirical attenuation equation, but still of the same order of magnitude.

The origin of the variability in the PGV residuals is further investigated by examining residuals for each station separately. Figure 10 illustrates how the variability at each distance range is composed by the interevent variability $\sigma_{e}$ (i.e., the variability at a single receiver due to different 

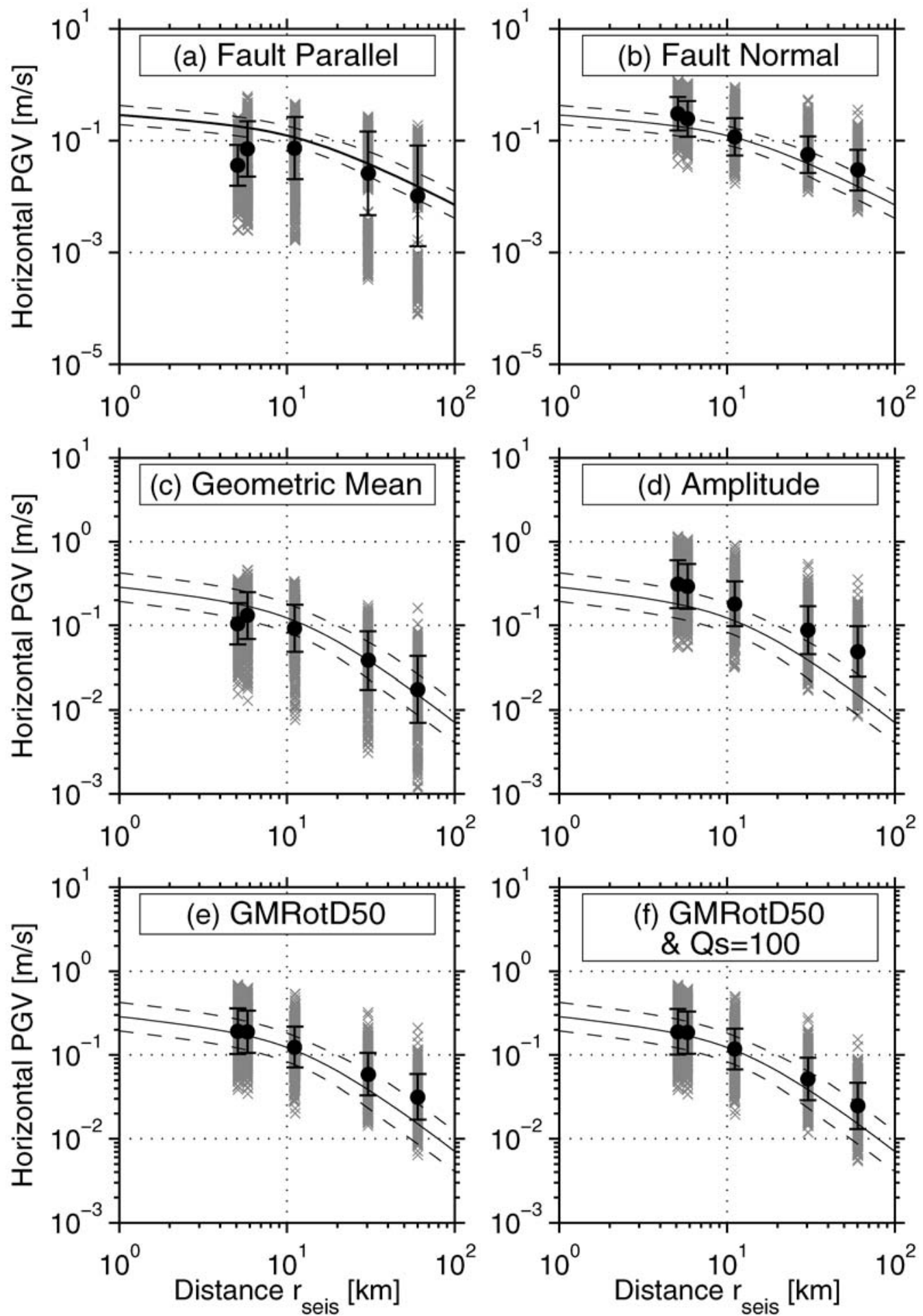

Figure 8. Comparison of PGV values of all 61 events of Figure 1 with empirical attenuation relation. The individual PGV values are plotted as gray crosses; their mean and standard deviation are indicated by the black circles and the error bars, respectively. Solid and dashed lines display the attenuation relation of Campbell (1997) and its 1- $\sigma$ bounds for an $M_{\mathrm{w}} 6.8$ event on a hard-rock site. (a) Fault-parallel component, (b) fault-normal component, (c) geometric mean of both horizontal PGV values, (d) PGV values of vector sum of the two horizontal components, (e) rotation independent geometric mean of PGV values as defined by Boore et al. (2006), and (f) same as (e) with $Q_{S}=100$ 

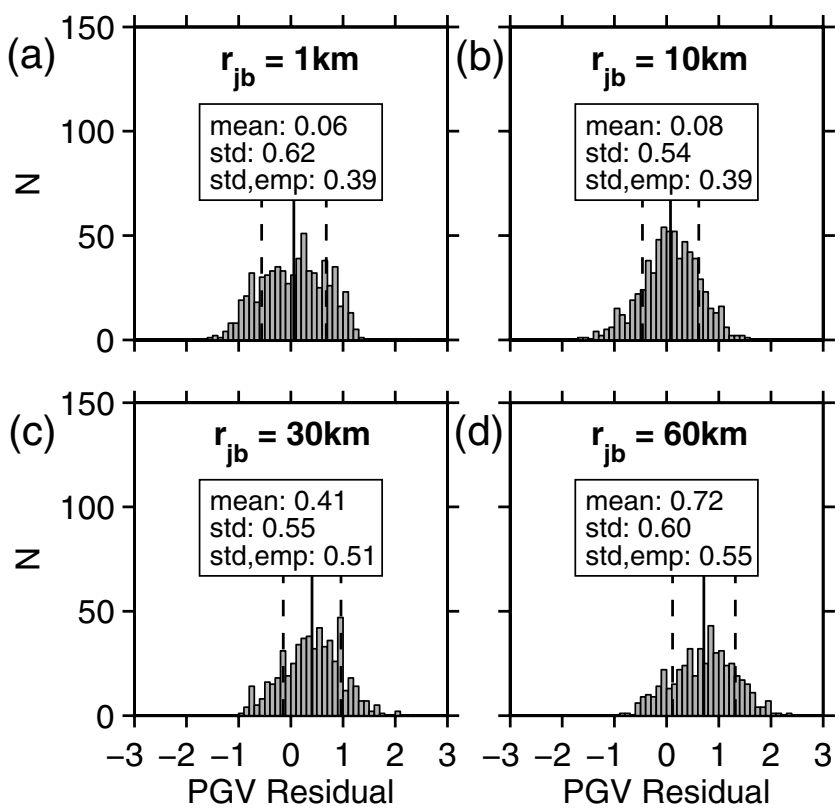

Figure 9. Distribution of residuals $\ln \left(\mathrm{PGV}_{\mathrm{syn}} / \mathrm{PGV}_{\mathrm{emp}}\right)$ of horizontal PGV (GMRotD50) at each distance range for all 61 events of Figure 1. Mean and standard deviation of the residuals are indicated by solid and dashed black lines, respectively. For comparison, the corresponding standard error estimate of the empirical attenuation relation by Campbell (1997) is specified as std,emp.

events) and the intraevent variability $\sigma_{a}$ (i.e., the variability for a single event considering all receivers at about the same distance). The interevent variability is computed for each receiver as the standard deviation of the residuals of the different events. The values given in Table 4 as $\sigma_{e}$ for each distance are the average interevent variabilities at all receivers of one distance range. The intraevent variability is calculated separately for each event and each distance range as the standard deviation of the residuals at all receivers in this distance range. By averaging these intraevent variabilities over all events we obtain the values of $\sigma_{a}$ for each distance range presented in Table 4. As a general trend, the interevent standard deviation $\sigma_{e}$ of the residuals is lowest in the azimuthal range around $90^{\circ}$, that is, perpendicular to the fault plane from the hypocenter. This is the region essentially unaffected by directivity, and thus, the variability here largely represents the differences in the rupture process due to the stress heterogeneity and the average stress level. Apart from the distance range of $1 \mathrm{~km}$, where the standard deviation of the residuals is poorly defined at the central azimuthal range, the interevent variability shows values of roughly $\sigma_{e} \approx 0.30$. This is lower than the total standard deviation $\sigma_{r}$ for each distance range as estimated previously. As can be seen in Figure 10, the higher total $\sigma_{r}$ originates from two sources. First of all, the interevent variabilities are higher $\left(\sigma_{e} \approx\right.$ 0.6-0.7) at the lower and upper ends of the azimuthal range, that is, in the region, where a receiver may either be situated in a forward or backward directivity position. Secondly, the $S$-wave radiation pattern leads to intraevent variabilities
Table 4

PGV Residuals

\begin{tabular}{rcccc}
\hline$r_{j b}(\mathrm{~km})$ & $\mu_{r}$ & $\sigma_{r}$ & $\sigma_{e}$ & $\sigma_{a}$ \\
\hline \multicolumn{5}{c}{ 61 events, variable hypocenters: } \\
1 & 0.06 & 0.62 & 0.61 & 0.44 \\
10 & 0.08 & 0.54 & 0.50 & 0.41 \\
30 & 0.41 & 0.55 & 0.49 & 0.47 \\
60 & 0.72 & 0.60 & 0.52 & 0.54 \\
\multicolumn{5}{c}{. } \\
1 & -0.06 & 0.82 & 0.47 & 0.75 \\
10 & -0.06 & 0.68 & 0.31 & 0.65 \\
30 & 0.27 & 0.74 & 0.25 & 0.75 \\
60 & 0.57 & 0.81 & 0.25 & 0.84 \\
\hline
\end{tabular}

$\mu_{r}$ and $\sigma_{r}$ are mean and standard deviation of residuals, respectively, for all receivers at the given distance. $\sigma_{e}$ and $\sigma_{a}$ are the average inter- and intraevent standard deviations, respectively. $\sigma_{e}$ is the average over all receivers at a given distance, while $\sigma_{a}$ is the average over all events.

$\sigma_{a} \approx 0.5$ in the same range as the average interevent variability. This is reflected by the differences in the mean PGV value along a receiver ring, showing up in Figure 10 as the distinct $\mathrm{W}$ shape of the average residual curve (higher residuals in the regions of $0^{\circ}, 90^{\circ}$, and $180^{\circ}$ and lower values in between), especially at larger distances.

We can further focus on the variability originating from the stress heterogeneity by analyzing the 30 model runs with fixed hypocenters. With identical stochastic stress parameters $\left(H=0.5, a_{c}=5 \mathrm{~km}\right.$, and S.D. $\left.=2 \mathrm{MPa}\right)$ and fixed hypocenters at $x=-10, z=15$, they are forced to have approximately the same unilateral rupture propagation. The remaining interevent variability of the ground-motion estimates therefore originates purely from the random nature of the stress field.

Figure 11 presents the PGV residuals at each receiver ring for these 30 simulations. Several observations can be made. First of all, the interevent variability $\sigma_{e}$ at each single receiver is lower than the total standard deviation for the whole suite of simulations, which is expected because of the forced similarity of the rupture process.

Second, the average interevent variability of the residuals decreases from $\sigma_{e} \approx 0.48$ at $r_{j b}=1 \mathrm{~km}$ toward $\sigma_{e} \approx$ 0.24 at $r_{j b}=60 \mathrm{~km}$. This demonstrates that the influence of the random fault stress on the ground motion decreases with distance from the source. This is again the expected behavior: distant stations experience an integrated effect of the rupture process, while near-fault stations are sensitive to local rupture complexities. Third, the largest variability for a single station within a given distance range is always observed in the backward directivity region whereas the smallest variability is always observed in the forward directivity region. This indicates that the directivity pulse due to the forced unilateral rupture propagation dominates the PGV estimate in the forward direction, while the differences in the rupture process are more likely to show up in the PGV measure in the backward direction. 

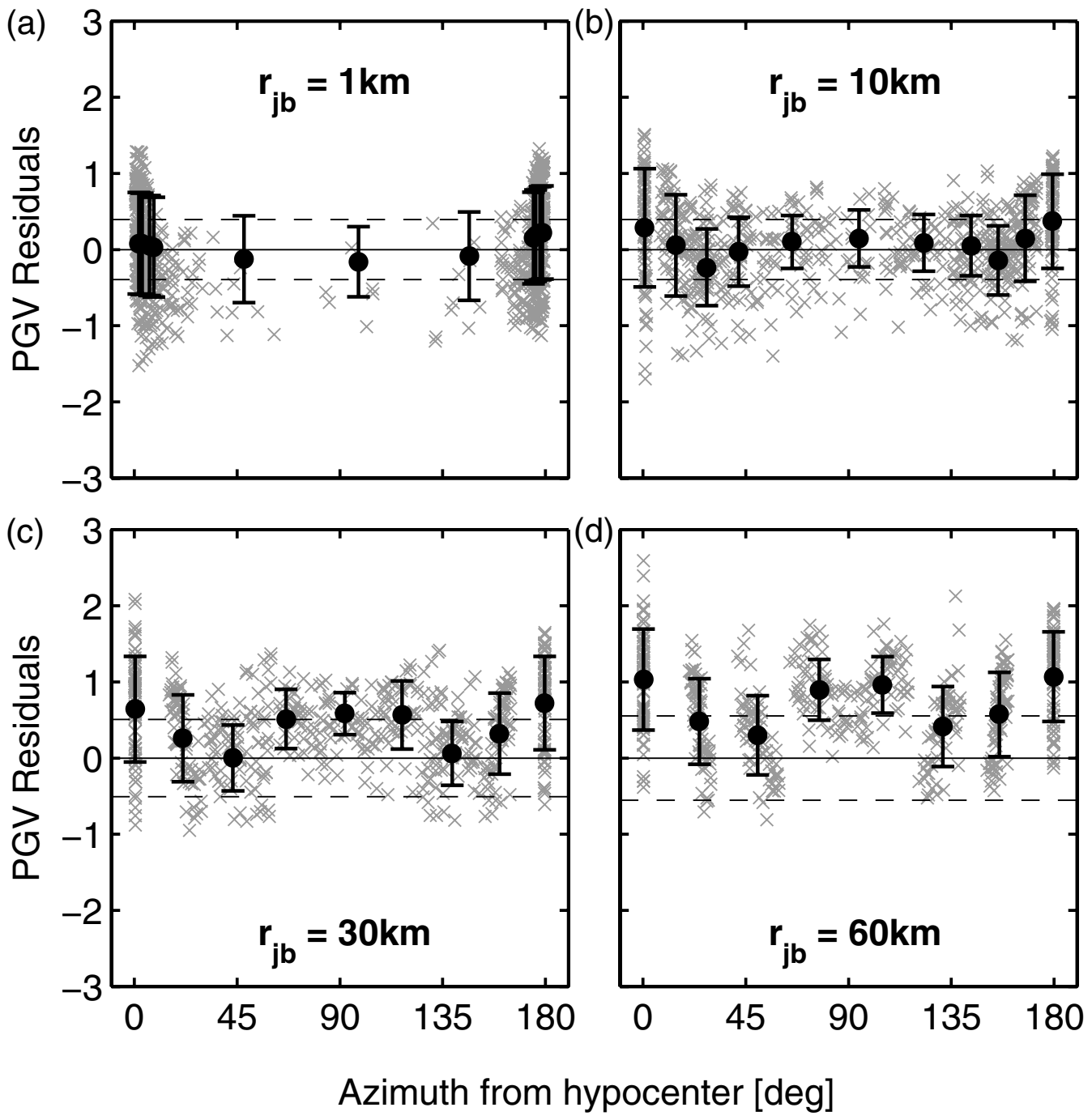

Figure 10. PGV residuals for single stations along receiver rings $\left(r_{j b} \approx 1,10,30\right.$, and $\left.60 \mathrm{~km}\right)$ for all 61 events of Figure 1 . Residual values are plotted in gray, their mean and standard deviation are indicated by black circles and error bars, respectively. Dashed horizontal lines specify the average standard deviation of the empirical relation for each distance range.

Finally, average residuals vary strongly with azimuth, clearly reflecting the directivity pattern. The resulting intraevent variability of $\sigma_{a} \approx 0.7-0.8$ is dominating the overall variability of a distance range $\left(\sigma_{r} \approx 0.7-0.8\right)$.

\section{Spectral Acceleration}

SA at different periods is a widely used parameter to quantify ground-motion intensity in seismic hazard analysis and engineering seismology. In the following we compare SA estimates of our synthetic ground motions with empirical attenuation relations derived by Campbell and Bozorgnia (2003). The choice of this particular set among other published attenuation relations (e.g., Abrahamson and Silva, 1997; Ambraseys et al., 2005) was motivated by the separate treatment of the firm-rock site class (same as hard rock in Campbell [1997]), which we consider more appropriate to compare with our assumed homogeneous half-space than the generic rock class used by most other attenuation relations.

At short distances, the SA relation for firm rock by Campbell and Bozorgnia (2003) is similar to the relation by Abrahamson and Silva (1997) for generic rock, but it decays faster at larger distances. Detailed comparisons between different SA attenuation relations can be found, for example, in Campbell and Bozorgnia (2003).

The computation of SA values for our synthetic seismograms is done by differentiating the ground velocity time histories to obtain acceleration traces and subsequently using the method of Newmark (e.g., see Chopra, 2001) to compute SA values for periods of $T=0.4,0.5,1$, and $4 \mathrm{sec}$ with a damping coefficient of $\zeta=5 \%$. The empirical attenuation relations by Campbell and Bozorgnia (2003) provide SA as a function of distance to the seismogenic rupture plane 

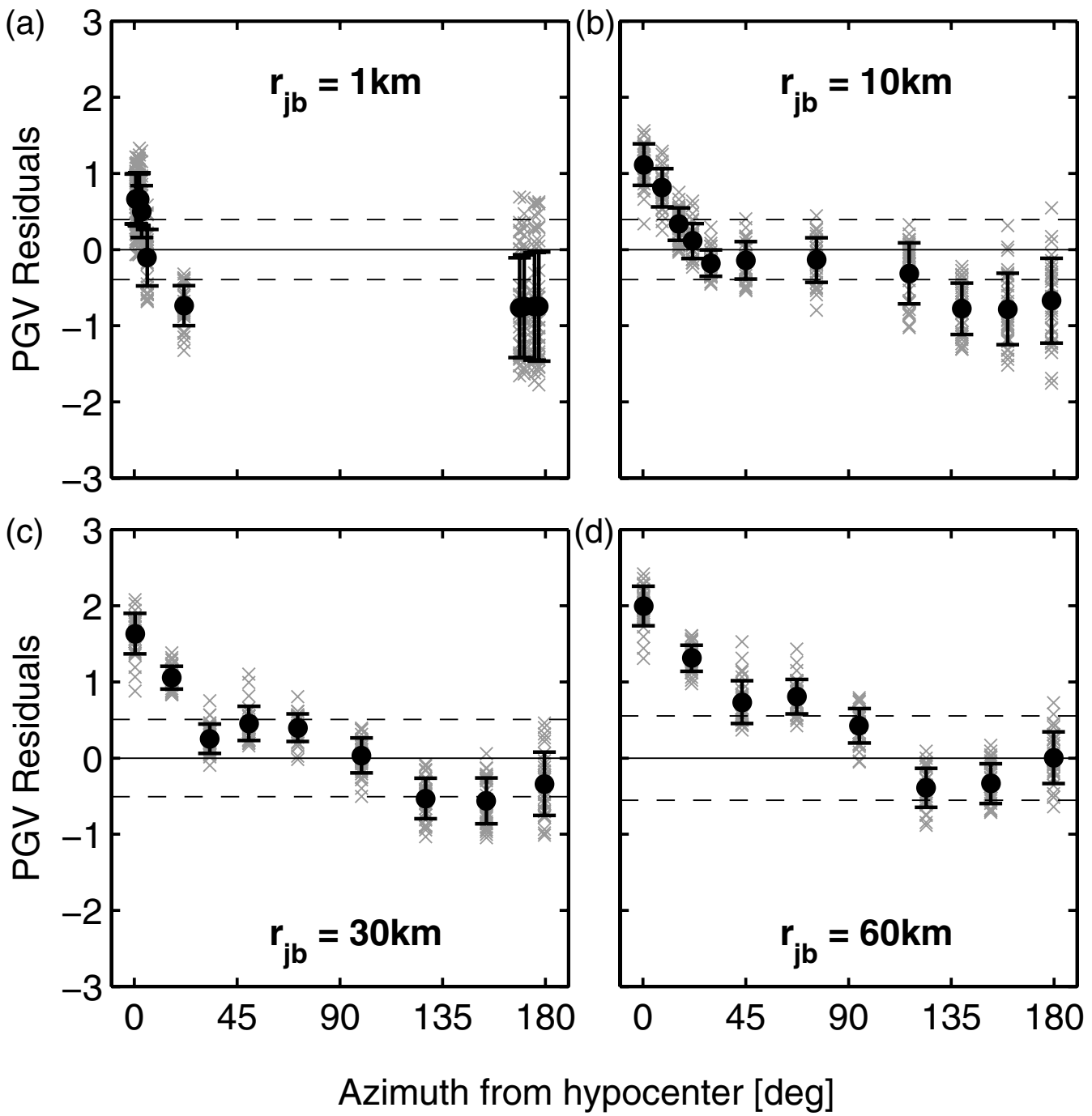

Figure 11. Same as Figure 10 but for the 30 simulations with fixed hypocenters. The directivity effect clearly shows up as high positive residuals at small azimuths (i.e., in the forward directivity region) and negative residuals at large azimuths (i.e., in the backward directivity region). The interevent variability at each station is smaller compared to Figure 10.

$r_{\text {seis }}$. As additional parameters for the attenuation equation we assume an $M_{\mathrm{w}} 6.8$ strike-slip event and set the appropriate factors for the firm-rock site conditions. The comparison for the four different periods is shown in Figure 12.

Our SA values are consistently lower than predicted by the empirical relation. The differences are largest for $T=0.4 \mathrm{sec}$ and decrease toward longer periods until at $T=$ $4 \mathrm{sec}$ the empirical relation is well matched at the farthest receivers. The fall-off rate with distance is generally in good agreement with the empirical relation, the exception being the nearest receivers (1- and 3-km distance to the surface projection of the fault) at the longest period of $T=4 \mathrm{sec}$.

We analyzed the variability in the SA residuals in the same way as for the PGV variability. In analogy to equation (4) we define the SA residuals as $r^{\mathrm{SA}}=\ln \left(\mathrm{SA}_{\mathrm{syn}}\right)-$ $\ln \left(\mathrm{SA}_{\mathrm{emp}}\right)$. For the period of $T=1 \mathrm{sec}$, Figure 13 illustrates the distribution of the residuals for different distances, and Table 5 summarizes their variabilities.
In general, the spatial distribution of the variability and its inter- and intraevent components are very similar to the values obtained for PGV.

PGV Variability of the 2004, Parkfield Earthquake

\section{Intraevent Variability}

The large number of ground-motion histories recorded close to the fault during the 2004, Parkfield, California, earthquake make this event a prime candidate for an estimation of the intraevent variability. However, because most of the Parkfield stations have a local site geology classified as soil, a direct comparison to our synthetics obtained for a homogeneous half-space is not possible. Furthermore, the $M_{\mathrm{w}}$ 6.0 Parkfield event was smaller than our simulated events, and rupture in the Parkfield event reached the surface, whereas our simulations assume a buried rupture. Neverthe- 

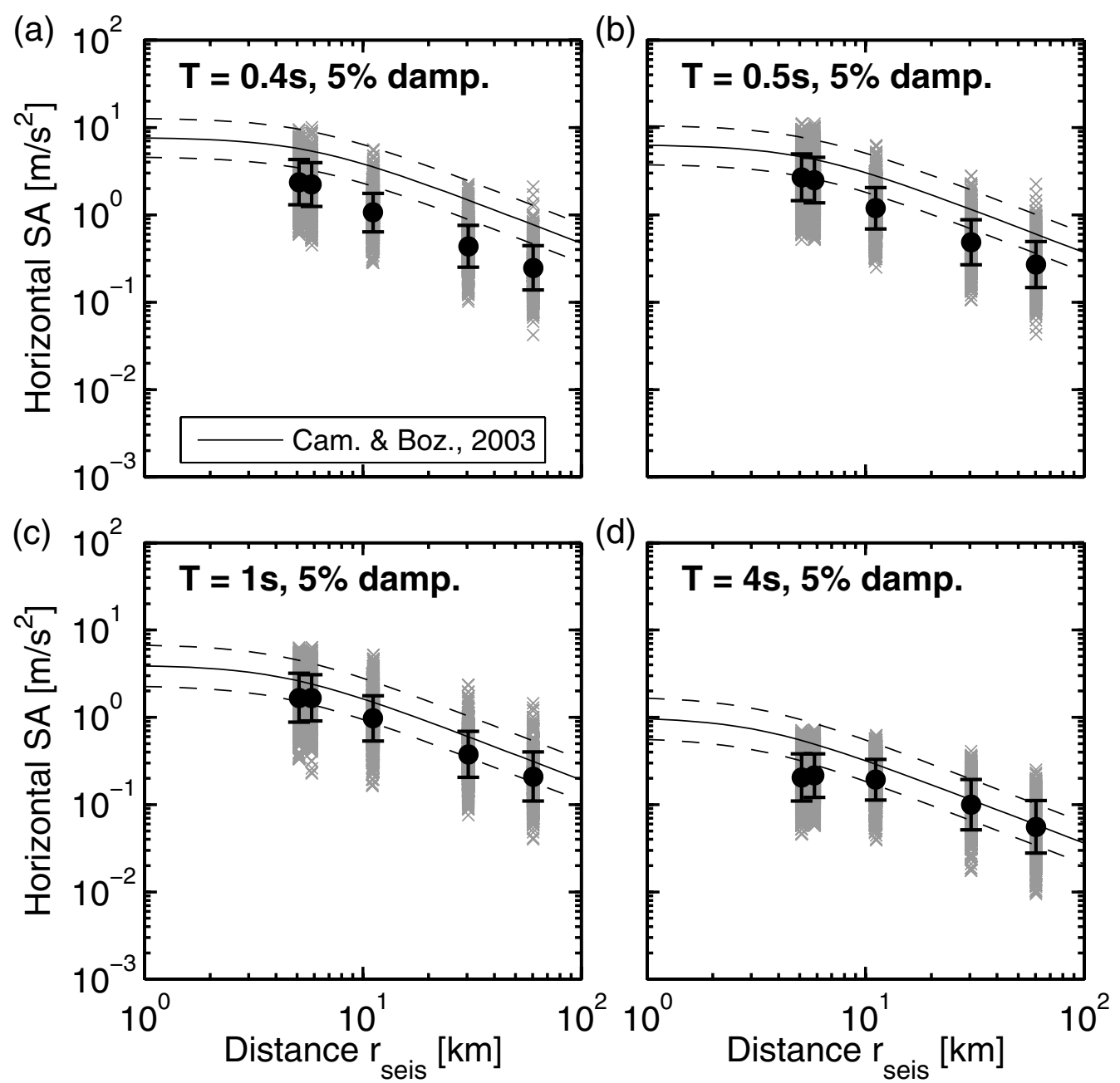

Figure 12. Comparison of horizontal SA for all 61 events of Figure 1 with empirical attenuation relation for periods of $0.4,0.5,1$, and $4 \mathrm{sec}$. The individual SA values (GMRotD50, see text) are plotted as gray crosses; their mean and standard deviation are indicated by black circles and error bars, respectively. Solid and dashed lines display the attenuation relation of Campbell and Bozorgnia (2003) and its 1- $\sigma$ bounds for an $M_{\mathrm{w}} 6.8$ event on a firm-rock site.

less, the Parkfield recordings may serve as an order-ofmagnitude estimate of the intraevent variability of peak ground motion in the near-fault region of large strike-slip earthquakes.

In a first step we make use of the PGV and distance data provided in table 1 of Shakal et al. (2006). There, PGV is given for each station as the larger of the east-west or north-south components, and distance is expressed as the closest distance to the fault surface trace. From the 95 stations listed in the table we selected all stations within the first $10 \mathrm{~km}$ from the fault, as station coverage farther out is too sparse for an estimation of the variability. We excluded the stations associated with buildings and also fault-zone 16 for which no PGV value was specified. These criteria yielded a set of 58 stations for further analysis. To make the estimation of the standard deviation more reliable, the variability at each distance $r$ from the fault is evaluated for all stations within a distance bin given by $r \pm 2 \mathrm{~km}$. The PGV intraevent variability was calculated as the standard deviation of the loga- rithm of the PGV values in each distance bin and is displayed in Figure 14a. From 0 to $7 \mathrm{~km}$ the variability is roughly constant at values between 0.60 and 0.65 . The apparent strong decrease at distances larger than $7 \mathrm{~km}$ can be largely attributed to the 12 stations of the U.S. Geological Survey Parkfield Dense Seismograph Array (UPSAR; Fletcher et al., 1992, 2006) that are located within a radius of about $1 \mathrm{~km}$ from each other at a distance of roughly $9 \mathrm{~km}$ from the fault. If all of the array stations apart from the central one are excluded (Fig. 14a), the variability continues on the same level out to $10-\mathrm{km}$ distance.

The analysis described in the preceding discussion used data from all available stations, irrespective of their local site geology. In a second step we therefore estimate the variability of ground motion within a single site class. For a comparison to our synthetic results, the rock site class would be the most interesting, but only the stations classified as soil are available in sufficiently large numbers for a statistical quantification. The geological classification for the stations of the 

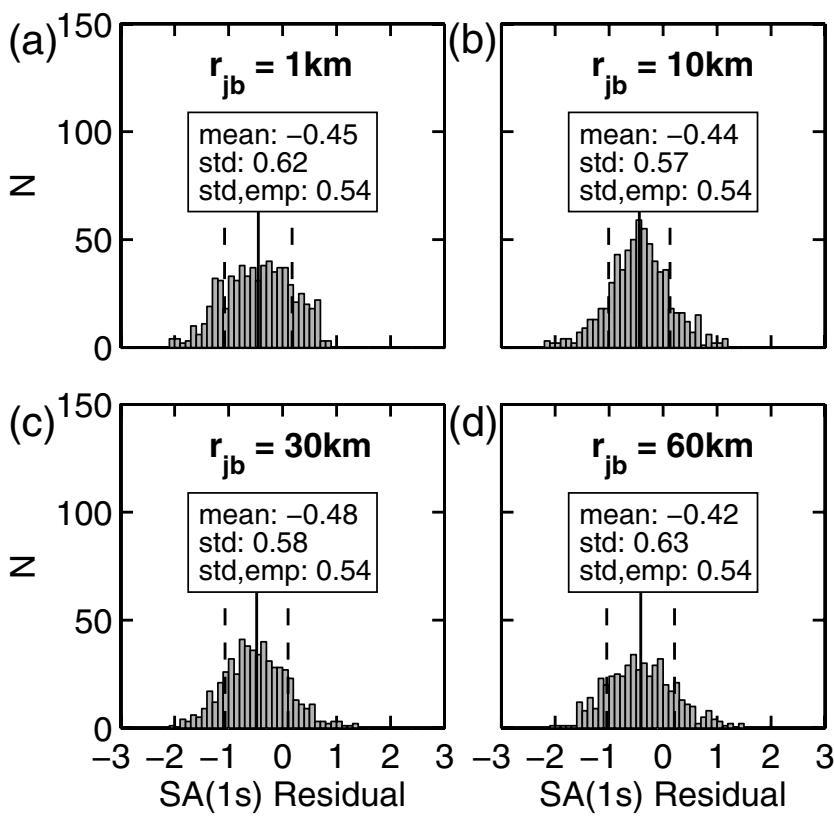

Figure 13. Distribution of residuals $\ln \left(\mathrm{SA}_{\mathrm{syn}} / \mathrm{SA}_{\mathrm{emp}}\right)$ for horizontal SA (GMRotD50, $T=1 \mathrm{sec}, 5 \%$ damping) at each distance range for all 61 events of Figure 1. Mean and standard deviation of the residuals are indicated by solid and dashed black lines, respectively. For comparison, the corresponding standard error estimate of the empirical attenuation relation by Campbell and Bozorgnia (2003) is specified as std,emp.

California Strong Motion Instrumentation Program (CSMIP) was taken from the web site of the Consortium of Organizations for Strong Motion Observation Systems (COSMOS, http://www.cosmos-eq.org, last accessed January 2007) but was not available for the U.S. Geological Survey (USGS) stations. From the 58 stations of the previous analysis we again exclude the UPSAR array stations apart from the central one. Of the remaining 47 stations, 22 are classified as soil stations. Figure $14 \mathrm{~b}$ shows the intraevent variability for these stations. The number of stations per distance bin is much lower than before, making these estimates less reliable. One curve displays the variability for the larger horizontal component as before, while the other is obtained for the GMRotD50 of PGV (Boore et al., 2006) calculated from the velocity waveforms. For this we used the corrected velocity traces as provided in the COSMOS database. To use the same frequency band as in our synthetics, the analysis was repeated with seismograms low-pass filtered at $2.5 \mathrm{~Hz}$ using a fourth order causal Butterworth filter (Fig. 14c). Because of this filtering the PGV estimates are lowered by $\sim 20 \%$ on average, which results in an average increase of the variabilities by $15 \%$ in the case of GMRotD50 and 24\% when using the larger horizontal component. For our simulated velocity seismograms we found PGV intraevent variabilities within the first $10 \mathrm{~km}$ of $\sim 0.4$ for variable hypocenter positions and roughly $0.7-0.8$ for unilaterally propagating ruptures (Table 4). Because the 2004 Parkfield, California, earthquake propagated mainly unilaterally, the PGV intraevent variabilities
Table 5

SA Residuals for the Period $T=1 \mathrm{sec}$

\begin{tabular}{rcccc}
\hline$r_{j b}(\mathrm{~km})$ & \multicolumn{1}{c}{$\mu_{r}$} & $\sigma_{r}$ & $\sigma_{e}$ & $\sigma_{a}$ \\
\hline \multicolumn{5}{c}{ 61 events, variable hypocenters: } \\
1 & -0.45 & 0.62 & 0.62 & 0.43 \\
10 & -0.44 & 0.57 & 0.52 & 0.46 \\
30 & -0.48 & 0.58 & 0.50 & 0.54 \\
60 & -0.42 & 0.63 & 0.53 & 0.59 \\
& 30 events, fixed hypocenters: & \\
1 & -0.57 & 0.80 & 0.53 & 0.71 \\
10 & -0.62 & 0.69 & 0.40 & 0.67 \\
30 & -0.62 & 0.79 & 0.37 & 0.80 \\
60 & -0.57 & 0.81 & 0.37 & 0.83
\end{tabular}

$\mu_{r}$ and $\sigma_{r}$ are the mean and standard deviation of residuals, respectively, for all receivers at the given distance. $\sigma_{e}$ and $\sigma_{a}$ are the inter- and intraevent standard deviations for each distance range. $\sigma_{e}$ is averaged over the receivers, while $\sigma_{a}$ is the average value over all events.

determined for the Parkfield recordings are in the same range as the variabilities obtained for our simulated velocity seismograms.

\section{Spatial Correlation of PGV}

The spatial variability of PGV of the Parkfield earthquake was analyzed by Shakal et al. (2006) using the approach described by Boore et al. (2003), essentially characterizing the spatial correlation of peak ground motion by studying how its variability is changing with increasing interstation distance. In the following we will perform the same analysis for our synthetic data. It consists of the following steps:

1. For all possible station pairs (1225), the interstation distance $\Delta$ is calculated.

2. For each pair, the difference between the logarithms of the PGV value is calculated, after accounting for the different fault distances. The latter correction is done using the PGV attenuation relation of Campbell (1997). Thus the difference in $\ln (\mathrm{PGV})$ is calculated as

$$
\Delta \ln \mathrm{PGV}=\left|\ln \left(\mathrm{PGV}_{1}\right)-\ln \left(\mathrm{PGV}_{2}\right) \frac{\ln \left[\mathrm{PGV}_{\mathrm{emp}}\left(r_{1}\right)\right]}{\ln \left[\mathrm{PGV}_{\mathrm{emp}}\left(r_{2}\right)\right]}\right|,
$$

where $\mathrm{PGV}_{1,2}$ are the simulated PGV values at two stations and $\mathrm{PGV}_{\mathrm{emp}}\left(r_{1,2}\right)$ are the empirical PGV estimates of Campbell (1997) for the distances $r_{1,2}$ of the two stations from the fault.

3. The station pairs are sorted by interstation distance in ascending order.

4. The standard deviation $\sigma_{\Delta \ln \text { PGV }}$ of $\Delta \ln$ PGV is calculated for each set of 15 consecutive station pairs.

5. The value of the calculated standard deviation is plotted versus the median value of the interstation distance of the 15 pairs. 

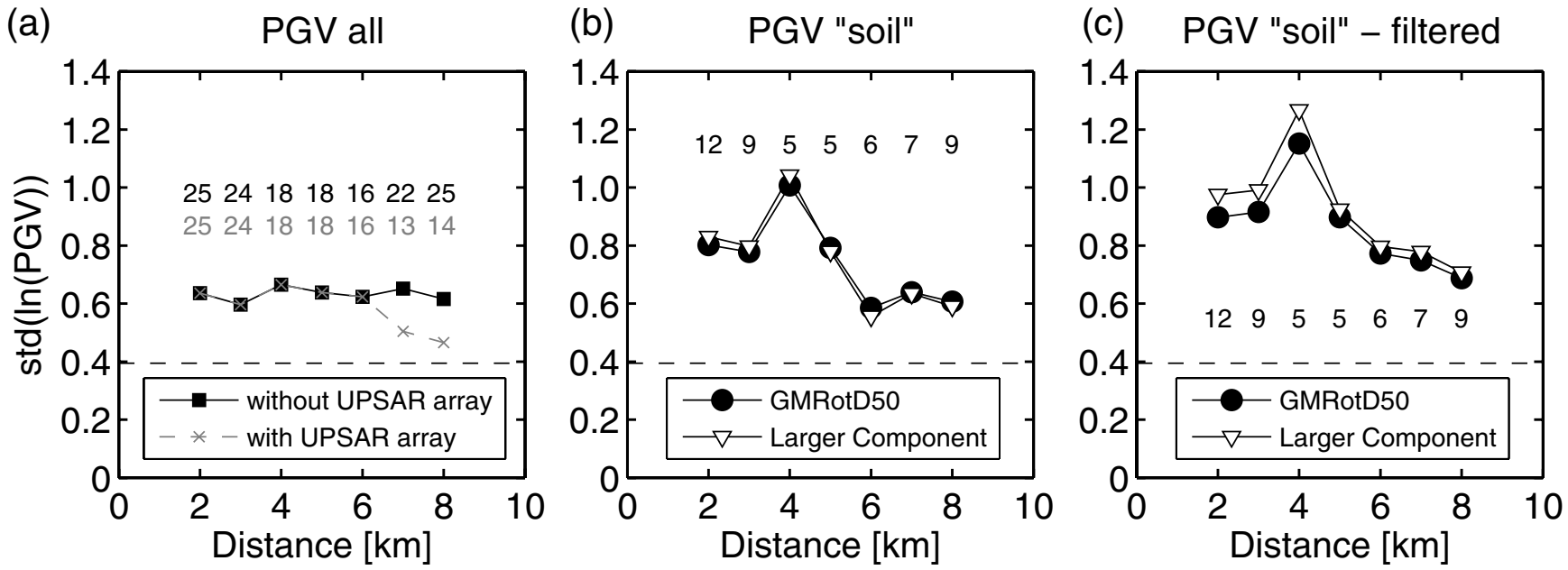

Figure 14. PGV variability of the $M_{\mathrm{w}} 6.0,2001$ Parkfield event. (a) Intraevent variability of PGV obtained from the data given in table 1 of Shakal et al. (2006). Stations with different local site conditions are included. The values above the symbols denote the number of stations per distance bin. The black curve was obtained by excluding 11 of the 12 UPSAR array stations, which are located close to each other at roughly 9-km distance from the fault. (b) Similar analysis for stations with local geology classified as soil, that is, for a subset of the stations used in the previous analysis. Numbers above the symbols denote the number of stations per distance bin. (c) Same as (b), but for seismograms lowpass filtered with a cutoff frequency of $2.5 \mathrm{~Hz}$.

To model the variation of $\sigma_{\Delta \ln \text { PGV }}$ with interstation distance $\Delta$, a relationship of the form

$$
\sigma_{\Delta \ln \text { PGV }}=\sigma_{\text {indobs }} \sqrt{1+\frac{1}{N}} F(\Delta)
$$

was used by Boore et al. (2003), where $\sigma_{\text {indobs }}$ denotes the standard deviation of an individual observation about a regression. As long as pairs of stations are considered, $N=1$ (D. M. Boore, personal comm., 2007) and $\sigma_{\text {indobs }} \sqrt{2}$ is the variability reached asymptotically at large interstation distances. The function $F(\Delta)$ defines the spatial correlation and is given by Boore et al. (2003) as

$$
F(\Delta)=1-\exp (-\sqrt{C \Delta}),
$$

where $C$ is a constant inversely related to a spatial correlation length. The PGA data of the 1994 Northridge earthquake was approximately fit by using $C=0.6$ and $\sigma_{\text {indobs }}=0.188$ (Boore et al., 2003). The PGV data of the 2004 Parkfield earthquake were shown to be consistent with $C=0.6$ and $\sigma_{\text {indobs }} \approx 0.24$ (values estimated by graphically matching figure 20 of Shakal et al. [2006]).

We computed regressions on our synthetic data (Fig. 15) assuming a functional form of equation (5) with $N=1$. The curves are least-squares fits over the range from 0 to $30 \mathrm{~km}$ with the two free parameters $\sigma_{\text {indobs }}$ and $C$. The obtained regression curves are displayed in Figure 15 for the 61 models with variable hypocenters and the 30 models with fixed hypocenters separately. Added to the plots in Figure 15 is a regression on the median values and the curves of Boore et al. (2003) and Shakal et al. (2006) with the values specified previously.
For the 61 events with variable hypocenters the regression on the median values yields $\sigma_{\text {indobs }}=0.30$ and $C=0.081$. For the 30 runs with fixed hypocenters we obtain values of $\sigma_{\text {indobs }}=0.74$ and $C=0.012$. The obtained values for $\sigma_{\text {indobs }}$ roughly match the average intraevent variabilities estimated in the section PGV Variability (Table 4) but are higher than the value found by Shakal et al. (2006) for the Parkfield earthquake. Our generally lower values of $C$ indicate longer correlation lengths than estimated by Boore et al. (2003) and Shakal et al. (2006).

\section{Discussion}

Directivity generally plays an important role in nearfield ground motions. Somerville et al. (1997) proposed empirically derived modifications based on the hypocenterreceiver geometry to incorporate the effects of directivity into SA attenuation relations. We applied these modifications to the attenuation relationship of Campbell and Bozorgnia (2003) and repeated the calculation of the SA residuals. The newly obtained residuals are smaller, that is, the SA values are closer to the empirical relation and their overall variability is reduced, thus confirming that the modifications of Somerville et al. (1997) capture the basic features of rupture directivity correctly. However, the achieved improvements are generally minor (e.g., $0.01-0.04 \log$ units for both $\mu_{r}$ and $\sigma_{r}$ at a 1 -sec period) and cannot account for the total discrepancies between SA of simulated and empirical groundmotion estimates.

The prominence of directivity effects in our simulations indicates that the seismic energy is radiated coherently despite some small-scale heterogeneity in the initial shear stress. As noted already by Ripperger et al. (2007), the 

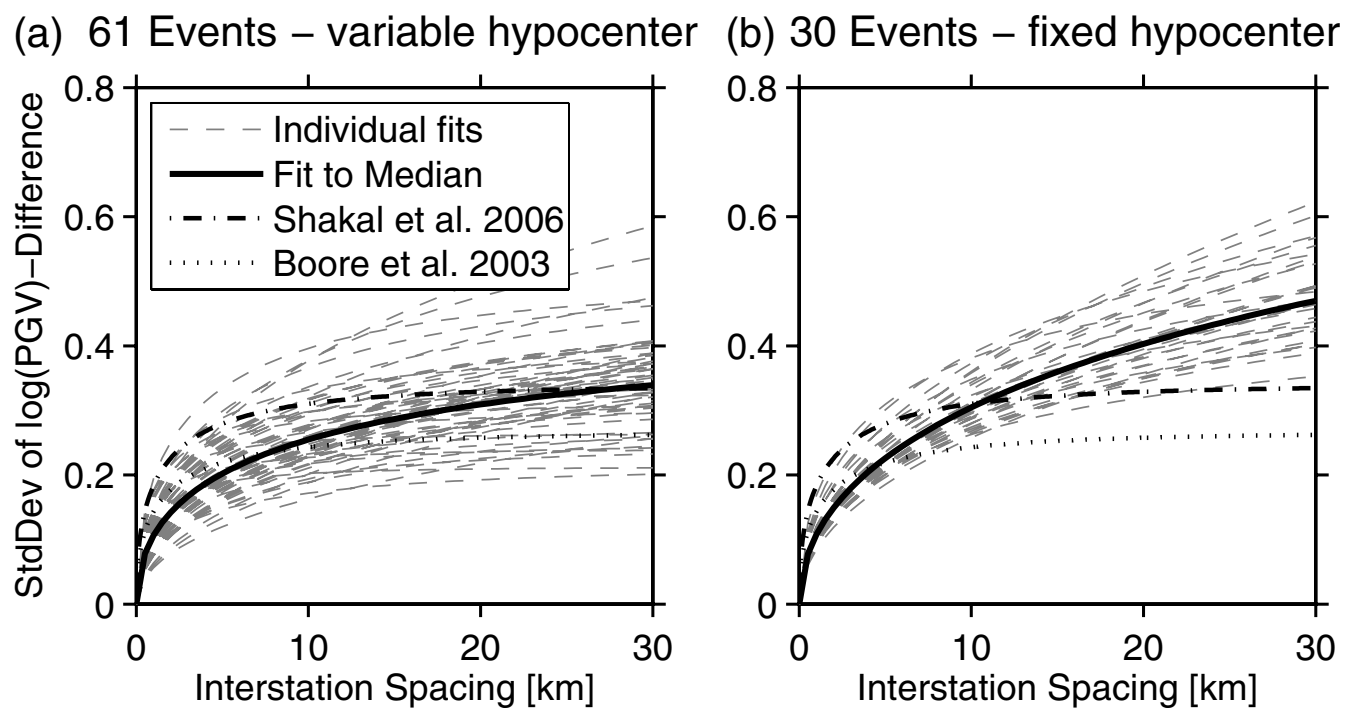

Figure 15. Regressions on standard deviation of the difference of $\ln (\mathrm{PGV})$ for all station pairs versus interstation distance (a) for the 61 events with variable hypocenters and (b) for the 30 events with fixed hypocenters. The solid black line represents the fit to the median values. The dash-dotted line reproduces the curve of Shakal et al. (2006, figure 20) fitting the PGV data of the 2004 Parkfield earthquake. Our analysis used the GMRotD50 value of PGV instead of the larger horizontal component as in Shakal et al. (2006). Finally the stippled line reproduces the curve presented in Boore et al. (2003, figure A1) to fit the PGA data for the 1994 Northridge earthquake.

presence of singular concentrations in the initial stress and heterogeneity in fracture energy are expected to be more efficient in generating highly variable rupture velocity and lead to incoherent radiation.

Nevertheless the random nature of the stress field leads to variability in the ground motion, which in many cases is superseded by the intraevent variability originating from the radiation pattern and directivity. Averaged over many events with different propagation directions, the total standard deviation of the residuals is on the order of $\sigma_{r} \approx 0.55-0.65$. This is in accord with the results by Aochi and Douglas (2006), who found a scatter in the simulations of $\sigma_{r} \approx 0.46-0.69\left(0.2-0.3\right.$ in $\log _{10}$ units) irrespective of the chosen scenario. Their simulations model rupture dynamics, but with completely homogeneous stress and friction parameters and for only one event for each scenario; hence, their scatter represents only the intraevent variability.

These values of the intraevent variability and also some of those obtained for our simulations may appear high and are in fact sometimes larger than standard deviations given in empirical attenuation relations. However, a high PGV intraevent variability of $\sigma_{a} \approx 0.6$ was obtained for the complete set of stations of the 2004 Parkfield earthquake. It is attributed to the mainly unidirectional nature of the rupture process. The even higher variabilities of $\sigma_{a} \approx 0.7-0.9$ obtained for the subset of soil stations may be partially biased by the small number of stations used for the analysis, but may also reflect a true increase in the variability due to strong effects of local site geology. These local site effects along with the 3D crustal structure of the Parkfield area are probably also responsible for the shorter spatial correlation lengths of the Parkfield ground-motion compared to our synthetics for which a homogeneous half-space has been assumed. Alternatively, our longer spatial correlation lengths may indicate an insufficient representation of real fault heterogeneity by our stress distributions. In this case, other statistical distributions of stress (apart from the Gaussian distribution assumed in the present study), the presence of singular concentrations in the initial stress, or an additional heterogeneity in fracture energy may be required.

The importance of the directivity effect has also been elucidated by studies involving kinematic source models (e.g., Aagaard et al., 2001). There the most important source parameters influencing the ground motion were found to be the depth of the fault, the rupture velocity, and the hypocenter-station geometry, and in particular the duration a rupture travels toward the observer.

Accordingly, one approach to predict the average ground-motion level and its variability could be to construct many kinematic models with varying fault depths, hypocenter positions, and rupture velocities. However, one would have to introduce some randomness in rupture velocity and the hypocenter position and possibly also in the slip and risetime distribution to do so. Hence, in the long run it may become the more natural and more physically constrained choice to quantify the uncertainty in the physical parameters such as stress and fracture energy as stochastic distributions on a given fault and do dynamic rupture simulations. These simulations will produce suites of models with varying hypocenters, rupture velocities, etc., as a result rather than an a priori input. However, at present there is no general consensus among scientists on the way dynamic models should be parameterized and in any of the models many of the input parameters are poorly constrained. Meanwhile, pseudody- 
namic modeling as proposed by Guatteri et al. (2004) is an intermediate approach that tries to improve the kinematic models by incorporating the most basic lessons learned from dynamic modeling.

\section{Conclusions}

We have computed synthetic near-field ground motion using a hybrid approach, where the rupture propagation is simulated by a boundary integral equation method (BIEM) and the wave-propagation is calculated using Green's functions from a discrete DWFE method.

The horizontal PGV estimates obtained from this approach are generally in good agreement with the empirically derived attenuation relation by Campbell (1997). Discrepancies are partially due to the computation method to obtain a single scalar PGV estimate from the two horizontal components. The use of an orientation-independent measure such as maximum vector amplitude or GMRotD50 as proposed by Boore et al. (2006) is therefore strongly suggested in future studies.

Compared to the empirical attenuation relation by Campbell and Bozorgnia (2003), the synthetic ground motions underestimate the SA at short periods and show an acceptable agreement at periods longer than $1 \mathrm{sec}$. We therefore conclude that at least for longer periods our modeling approach can be used to realistically simulate ground-motion characteristics of interest in engineering seismology and seismic hazard assessment.

The different factors contributing to ground-motion variability were separated by detailed analysis. We find that the ground-motion variability due to the stress heterogeneity is generally largest close to the fault and is largest in the backward directivity region for mostly unilateral rupture propagation. In all cases the intraevent variability due to directivity effects and the $S$-wave radiation pattern is on the order of or larger than the interevent variability. The contribution to the interevent variability from differences in the hypocenter-station configuration is found to be larger than the contribution resulting from differences in the heterogeneous initial stress. In other words, in our current model stress heterogeneity contributes more to ground-motion variability by determining the hypocenter location than it does by influencing the dynamic rupture process. However, the influence on the dynamic rupture process might become the more important contribution to ground-motion variability for a representation of fault heterogeneities that includes residual stress singularities and sharp fracture energy heterogeneities. An analysis of the ground-motion recordings of the 2004 Parkfield, California, earthquake reveals that the PGV intraevent variability of this event is comparable to the intraevent variabilities of our synthetics.

Overall, this study represents a step toward a physicsbased estimation of future ground-motion levels for purposes in seismic hazard assessment.

\section{Acknowledgments}

We are grateful to two anonymous reviewers and to the associate editor D. Oglesby for their comments and suggestions that helped to improve the manuscript. We thank E. Dunham, Harvard University, for providing his spectral boundary integral code. Banu Mena kindly supplied us with a script for the computation of spectral acceleration. J.-P. Ampuero is supported by SPICE, a Marie Curie Research Training Network in the 6th Framework Program of the European Commission.

\section{References}

Aagaard, B. T., J. F. Hall, and T. H. Heaton (2001). Characterization of nearsource ground motions with earthquake simulations, Earthq. Spectra 17, no. 2, 177-207.

Aagaard, B. T., J. F. Hall, and T. H. Heaton (2004). Effects of fault dip and slip rake angles on near-source ground motions: why rupture directivity was minimal in the 1999 Chi-Chi, Taiwan, earthquake, Bull. Seismol. Soc. Am. 94, no. 1, 155-170.

Abercrombie, R. E., and J. R. Rice (2005). Can observations of earthquake scaling constrain slip-weakening?, Geophys. J. Int. 162, 406-424, doi 10.1111/j.1365246X.2005.02579.x.

Abrahamson, N. A., and W. J. Silva (1997). Empirical response spectral attenuation relations for shallow crustal earthquakes, Seism. Res. Lett. 68, no. 1, 94-68.

Aki, K. (1968). Seismic displacements near a fault, J. Geophys. Res. 73, 5359-5376

Aki, K., and P. G. Richards (2002). Quantitative Seismology, Second Ed., University Science Books, Sausalito, California.

Ambraseys, N. N., J. Douglas, S. K. Sarma, and P. M. Smit (2005). Equations for the estimation of strong ground motions from shallow crustal earthquakes using data from Europe and the Middle East: horizontal peak ground acceleration and spectral acceleration, Bull. Earthq. Eng. 3, 1-53, doi 10.1007/s10518-005-0183-0.

Ampuero, J.-P., J. Ripperger, and P. M. Mai (2006). Properties of dynamic earthquake ruptures with heterogeneous stress drop, in Earthquakes: Radiated Energy and the Physics of Faulting, R. Abercrombie, A. McGarr and H. Kanamori (Editors), American Geophysical Monograph 170, 255-261.

Andrews, D. J. (1980). A stochastic fault model 1. Static case, J. Geophys. Res. 85, no. B7, 3867-3877.

Aochi, H., and J. Douglas (2006). Testing the validity of simulated strong ground motion from the dynamic rupture of a finite fault, by using empirical equations, Bull. Earthq. Eng. 4, 211-229, doi 10.1007/ s10518-006-0001-3.

Archuleta, R. J., and G. A. Frazier (1978). Three-dimensional numerical simulations of dynamic faulting in a half-space, Bull. Seismol. Soc. Am. 68, no. 3, 541-68.

Beyer, K., and J. J. Bommer (2006). Relationships between median values and between aleatory variabilities for different definitions of the horizontal component of motion, Bull. Seismol. Soc. Am. 96, no. 4A, $1512-1522$, doi 10.1785/0120050210.

Boatwright, J., K. Thywissen, and L. C. Seekins (2001). Correlation of ground motion and intensity for the 17 January 1994 Northridge, California, earthquake, Bull. Seismol. Soc. Am. 91, no. 4, 739-752.

Boore, D. M., W. B. Gibbs, W. B. Joyner, J. C. Tinsley, and D. J. Ponti (2003). Estimated ground motion from the 1994 Northridge, California, earthquake at the site of the Interstate 10 and La Cienega Boulevard bridge collapse, West Los Angeles, California, Bull. Seismol. Soc. Am. 93, no. 6, 2737-2751.

Boore, D. M., J. Watson-Lamprey, and N. A. Abrahamson (2006). Orientation independent measures of ground motion, Bull. Seismol. Soc. Am. 96, no. 4A, 1502-1511, doi 10.1785/0120050209.

Campbell, K. W. (1997). Empirical near-source attenuation relationships for horizontal and vertical components of peak ground acceleration, peak ground velocity, and pseudo absolute acceleration response spectra, Seism. Res. Lett. 68, no. 1, 154-179. 
Campbell, K. W. (2000). Erratum: "Empirical near-source attenuation relationships for horizontal and vertical components of peak ground acceleration, peak ground velocity, and pseudo-absolute acceleration response spectra", Seism. Res. Lett. 71, no. 3, 352-354.

Campbell, K. W. (2001). Erratum: "Empirical near-source attenuation relationships for horizontal and vertical components of peak ground acceleration, peak ground velocity, and pseudo-absolute acceleration response spectra", Seism. Res. Lett. 72, no. 4, 474.

Campbell, K. W., and Y. Bozorgnia (2003). Updated near-source groundmotion (attenuation) relations for the horizontal and vertical components of peak ground acceleration and acceleration response spectra, Bull. Seismol. Soc. Am. 93, no. 1, 314-331.

Chopra, A. K. (2001). Dynamics of structures: Theory and applications to earthquake engineering, International Series in Civil Engineering and Engineering Mechanics, Second Ed., Prentice Hall, New Jersey.

Das, S., and K. Aki (1977). A numerical study of two-dimensional spontaneous rupture propagation, Geophys. J. R. Astr. Soc. 50, 643-668.

Dunham, E. M. (2005). Dissipative interface waves and the transient response of a three dimensional sliding interface with Coulomb friction, J. Mech. Phys. Sol. 53, 327-357, doi 10.1016/j.jmps.2004.07.003.

Fletcher, J. B., L. M. Baker, P. Spudich, P. Goldstein, J. D. Sims, and M. Hellweg (1992). The USGS Parkfield, California, dense seismograph array: UPSAR, Bull. Seismol. Soc. Am. 82, no. 2, 1041-1070.

Fletcher, J. B., P. Spudich, and L. M. Baker (2006). Rupture propagation of the 2004 Parkfield, California, earthquake from observations at the UPSAR, Bull. Seismol. Soc. Am. 96, no. 4B, S129-S142, doi $10.1785 / 0120050812$

Futterman, W. I. (1962). Dispersive body waves, J. Geophys. Res. 67, no. 13, 5279-5291.

Geubelle, P. H., and J. R. Rice (1995). A spectral method for threedimensional elastodynamic fracture problems, J. Mech. Phys. Sol. 43, no. 11, 1791-1824.

Graves, R. W. (1998). Three-dimensional Finite-Difference modeling of the San Andreas fault: source parameterization and ground-motion levels, Bull. Seismol. Soc. Am. 88, no. 4, 881-897.

Graves, R., and A. Pitarka (2004). Broadband time history simulation using a hybrid approach, in Proceedings of the 13th World Conference on Earthquake Engineering, Vancouver, B.C., Canada, paper no. 1098.

Guatteri, M., P. M. Mai, and G. C. Beroza (2004). A pseudo-dynamic approximation to dynamic rupture models for strong ground motion prediction, Bull. Seismol. Soc. Am. 94, no. 6, 2051-2063.

Haskell, N. (1969). Elastic displacements in the near-field of a propagating fault, Bull. Seismol. Soc. Am. 59, no. 2, 865-908.

Inoue, T., and T. Miyatake (1998). 3D simulation of near-field strong ground motion based on dynamic modeling, Bull. Seismol. Soc. Am. 88, no. 6, $1445-1456$

Kanamori, H., and E. Brodsky (2004). The physics of earthquakes, Rep. Prog. Phys. 67, 1429-1496, doi 10.1088/0034-4885/67/8/R03.

Mai, P. M. (2004). SRCMOD: a database of finite-source rupture models, www.seismo.ethz.ch/srcmod (last accessed December 2006).

Mai, P. M., and G. C. Beroza (2000). Source scaling properties from finitefault-rupture models, Bull. Seismol. Soc. Am. 90, no. 3, 604-615.

Mai, P. M., and G. C. Beroza (2002). A spatial random field model to characterize complexity in earthquake slip, J. Geophys. Res. 107, no. B11, 2308, doi 10.1029/2001JB000588.

Mai, P. M., P. Somerville, A. Pitarka, L. Dalguer, H. Miyake, G. Beroza, S.-G. Song, and K. Irikura (2006). Fracture-energy scaling in dynamic rupture models of past earthquakes, in Earthquakes: Radiated Energy and the Physics of Faulting, R. Abercrombie, A. McGarr and H. Kanamori (Editors), American Geophysical Monograph, 170, 283-293.

Mai, P. M., P. Spudich, and J. Boatwright (2005). Hypocenter locations in finite-source rupture models, Bull. Seismol. Soc. Am. 95, no. 3, 965-980, doi 10.1785/0120040111.

Nielsen, S. B., and K. B. Olsen (2000). Constraints on stress and friction from dynamic rupture models of the 1994 Northridge, California, earthquake, Pure Appl. Geophys. 157, 2029-2046.
Oglesby, D. D., and S. M. Day (2002). Stochastic fault stress: implications for fault dynamics and ground motion, Bull. Seismol. Soc. Am. 92, no. $8,3006-3021$.

Oglesby, D. D., R. J. Archuleta, and S. B. Nielsen (2000). The threedimensional dynamics of dipping faults, Bull. Seismol. Soc. Am. 90, no. 3, 616-628.

Olsen, K. B., S. M. Day, J. B. Minster, Y. Cui, A. Chourasia, M. Faerman, R. Moore, P. Maechling, and T. Jordan (2006). Strong shaking in Los Angeles expected from southern San Andreas earthquake, Geophys. Res. Lett. 33, L07305, doi 10.1029/2005GL025472.

Olsen, K. B., R. Madariaga, and R. J. Archuleta (1997). Three-dimensional dynamic simulation of the 1992 Landers earthquake, Science 278, 834-838.

Olson, A. H., J. A. Orcutt, and G. A. Frazier (1984). The discrete wavenumber/finite element method for synthetic seismograms, Geophys. J. R. Astr. Soc. 77, 421-460.

Peyrat, S., and K. B. Olsen (2004). Nonlinear dynamic rupture inversion of the 2000 Western Tottori, Japan, earthquake, Geophys. Res. Lett. 31, L05604, doi 10.1029/2003GL019058.

Ripperger, J., and P. M. Mai (2004). Fast computation of static stress changes on 2D faults from final slip distributions, Geophys. Res. Lett. 31, L18610, doi 10.1029/2004GL020594.

Ripperger, J., J.-P. Ampuero, P. M. Mai, and D. Giardini (2007). Earthquake source characteristics from dynamic rupture with constrained stochastic fault stress, J. Geophys. Res 112, B04311, doi 10.1029/ 2006JB004515.

Shakal, A., H. Haddadi, V. Graizer, K. Lin, and M. Huang (2006). Some key features of the strong-motion data from the M 6.0 Parkfield, California, earthquake of 28 September 2004, Bull. Seismol. Soc. Am. 96, no. 4b, S90-S118, doi 10.1785/0120050817.

Somerville, P. G., N. F. Smith, R. W. Graves, and N. A. Abrahamson (1997). Modification of empirical strong ground motion attenuation relations to include the amplitude and duration effects of rupture directivity, Seism. Res. Lett. 68, no. 1, 199-222.

Spudich, P., and L. Xu (2003). Software package COMPSYN: programs for earthquake ground motion calculation using complete 1-D Green's functions, in International Handbook of Earthquake \& Engineering Seismology, in International Geophysics Series, W. H. K. Lee, H. Kanamori, P. Jennings and C. Kisslinger (Editors), Vol. 81, Academic Press, Amsterdam.

Wald, D. J., V. Quitoriano, T. H. Heaton, and H. Kanamori (1999). Relationships between peak ground acceleration, peak ground velocity and modified Mercalli intensity in California, Earthq. Spectra 15, no. 3, 557-564.

Wells, D. L., and K. J. Coppersmith (1994). New empirical relationships among magnitude, rupture length, rupture width, rupture area, and surface displacement, Bull. Seismol. Soc. Am. 84, no. 4, 974-1002.

\section{Appendix}

\section{Computation of Static Stress Loading}

\section{Problem Statement}

As mentioned in the main text of this article, the hypocenter location in our modeling approach is determined to be mechanically consistent with the stress heterogeneity. The two main assumptions underlying our approach are (1) a uniformly rising background stress driven by tectonic loading and (2) the validity of the slip-weakening friction law also during the slow, quasi-static nucleation process of the earthquake. The problem to be solved thus breaks down to finding the uniform increase in stress $\tau_{c}^{\infty}$ (critical load), which drives 
a given heterogeneous stress distribution $\tau_{0}(\underline{x})$ on a fault to the critical stage at the onset of dynamic instability and the associated preslip and stress distributions to be used as initial conditions in the dynamic rupture model. Once a point of the fault reaches the yield strength $\tau_{s}$ of the material, it is allowed to slip. Because the rising of stress is assumed to occur slowly, slip on the fault is assumed to take place quasistatically, but nevertheless obeying the linear slip-weakening friction law with sliding strength $\tau_{d}$ and critical slipweakening distance $D_{c}$. So for each infinitesimal increase in background stress $\tau^{\infty}$, the effects of the uniform increase; the stress change due to slip and finally the strength drop due to slip have to be considered.

\section{Efficient Solution}

The main idea for solving the task efficiently is to recognize that the incremental problem restricted to the subset of actively slipping points of the numerical grid, $I_{\nu}$, is a linear problem until $\tau_{S}$ is reached by a grid point outside $I_{\nu}$ or until $D_{c}$ is reached by a point inside $I_{\nu}$.

Inside the slipping zone $I_{\nu}$ the stress is exactly balanced by friction:

$$
\tau^{\infty}(t)+\tau_{0}(\underline{x})+\mathcal{K}[\delta]=\tau_{s}-W \delta,
$$

where $W$ is the slip-weakening rate $\left(\tau_{s}-\tau_{d}\right) / D_{c}$ and $\mathcal{K}[\delta]$ is the elastostatic stress distribution due to slip $\delta(\underline{x}, t)$. The operator $\mathcal{K}[\cdot]$ is linear. The incremental version is

$$
\Delta \tau^{\infty}+\mathcal{K}[\Delta \delta]=-W \Delta \delta \quad \text { inside } I_{\nu},
$$

where $\Delta$ refers to changes from a previous equilibrium state. After numerical discretization and rearranging one gets

$$
\Delta \tau^{\infty}+\left(K_{\nu}+W_{\nu}\right) \Delta \delta_{\nu}=0
$$

where $K_{\nu}$ and $\Delta \delta_{\nu}$ denote the static stiffness matrix and the slip increment, respectively, for the $N_{\nu}$ points belonging to $I_{\nu}$ and $W_{\nu}$ is a diagonal matrix of size $N_{\nu} \times N_{\nu}$ with diagonal value $W$, where $\delta<D_{c}$ and 0 elsewhere. As long as $I_{\nu}$ is fixed, (A3) is a linear system of $N_{\nu}$ equations with $N_{\nu}+1$ unknowns, $\Delta \tau^{\infty}$ and $\Delta \delta_{\nu}$. To obtain a well-defined (square) problem an additional constraint has to be appended. This can be done in various ways, but the following choice leads to a symmetric square problem:

$$
\Delta \delta_{\nu} \underline{1}=1
$$

In other terms, the value of the increment of seismic potency ( $\Delta$ potency $=\Delta x^{2}$ ) is prescribed. The complete linear problem is

$$
\left(\begin{array}{cc}
K_{\nu}+W_{\nu} & \underline{1} \\
\underline{1}^{T} & 0
\end{array}\right)\left(\begin{array}{c}
\Delta \delta_{\nu}^{*} \\
\Delta \tau_{\infty}^{*}
\end{array}\right)=\left(\begin{array}{c}
\underline{0} \\
1
\end{array}\right) .
$$

An * superscript has been added to highlight that the solution $\left(\Delta \delta^{*}, \Delta \tau_{\infty}^{*}\right)$ corresponds to an arbitrarily prescribed value of potency increment. This solution can be rescaled by an arbitrary multiplicative factor $\lambda$. The physical value of $\lambda$ is ultimately determined according to some additional constraint, in our case that the stress outside $I_{\nu}$ remains below $\tau_{s}$ for a positive $\Delta \tau^{\infty}$.

\section{The Algorithm}

The algorithm used for this article consists of the following steps:

1. Find the set of points $I_{\nu}$, where either (a) stress has reached $\tau_{s}\left(\tau \geq \tau_{s}\right)$ and/or (b) slip is already taking place $(\delta>0)$.

2. Build $K_{\nu}$, the static stiffness matrix for the set of slipping points $I_{\nu}$. It is a $N_{\nu} \times N_{\nu}$ matrix, where $N_{\nu}$ is the number of elements of $I_{\nu}$. This step involves computation of the static stress change due to a unit increase in slip at a single grid point. Here the algorithm makes use of the efficient spectral formulation discussed by Ripperger and Mai (2004).

3. Solve matrix equation (A5) for $\Delta \delta_{\nu}^{*}$ and $\Delta \tau_{\infty}^{*}$. This step could be performed by an efficient linear solver.

4. If $\Delta \tau_{\infty}^{*} \leq 0$, exit the algorithm and report the $\tau^{\infty}$ reached so far as the critical value $\tau_{c}^{\infty}$ and use the current stress and preslip distribution as input to the dynamic rupture calculation. Else go on with steps 5-7.

5. Compute the stress change $\Delta \tau^{*}$ for the increment $\Delta \tau_{\infty}^{*}$ of background stress, including the redistribution of stress due to slip:

$$
\Delta \tau^{*}=\Delta \tau_{\infty}^{*} \underline{1}+K \Delta \delta^{*}
$$

where now 1 is a $N \times 1$ column vector of ones (with $N$ being the total number of points in the grid), $K$ is the total $N \times N$ stiffness matrix, and $\Delta \delta^{*}$ is a $N \times 1$ vector with the values of $\Delta \delta_{\nu}^{*}$ at the points of $I_{\nu}$ and 0 everywhere else. Again, use is made of the spectral formulation of Ripperger and Mai (2004) relating static stress changes to a slip distribution. This circumvents the need to construct the large matrix $K$, but allows one to do the computation efficiently by employing the fast Fourier transform (FFT).

6. Determine the (scalar) multiplicative factor $\lambda$ from two additional constraints: The first constraint is that the new traction values $\tau_{\text {new }}=\tau+\lambda \Delta \tau^{*}$ outside $I_{\nu}$ must not exceed $\tau_{s}$, that is, $\tau_{\text {new }} \leq \tau_{s}$ :

$$
\lambda_{\text {stress }}=\min _{\oplus}\left[\frac{\tau_{s}-\tau}{\Delta \tau^{*}}\right]_{{\text {outside } I_{\nu}}} .
$$

Here the $\oplus$ indicates that the minimum is taken only of the positive values of the expression in brackets, whereas the negative values are ignored. The second constraint is 
that the new slip $\delta_{\text {new }}=\delta+\lambda \Delta \delta^{*}$ should not exceed $D_{c}$ in those points that have slip $\delta$ between $0<\delta<D_{c}$, that is,

$$
\lambda_{\text {slip }}=\min _{\oplus}\left[\frac{D_{c}-\delta}{\Delta \delta_{\nu}^{*}}\right]_{\text {inside }_{\nu}, \delta<D_{c}} .
$$

And for the actual factor $\lambda$ the minimum of the values given by equations (A7) and (A8) is taken.

7. (a) Update the values of stress and slip

$$
\tau_{\text {new }}=\tau+\lambda \Delta \tau^{*} \quad \delta_{\text {new }}=\delta+\lambda \Delta \delta^{*},
$$

(b) keep track of the total increase in background stress

$$
\tau_{\text {new }}^{\infty}=\tau^{\infty}+\lambda \Delta \tau_{\infty}^{*}
$$

(c) set the slip-weakening rate to $W=0$ for all points that now have $\delta \geq D_{c}$, and (d) finally start over at step 1 with the newly obtained values.

Institute of Geophysics

ETH Zurich, 8093 Zurich, Switzerland ripperger@sed.ethz.ch

Manuscript received 20 March 2007 